\title{
Ectopic Expression of the Neuropeptide Pigment-Dispersing Factor Alters Behavioral Rhythms in Drosophila melanogaster
}

\author{
Charlotte Helfrich-Förster, ${ }^{1}$ Marcus Täuber, ${ }^{2}$ Jae H. Park, ${ }^{3}$ Max Mühlig-Versen,, ${ }^{2}$ Stephan Schneuwly, ${ }^{2}$ and \\ Alois Hofbauer ${ }^{2}$ \\ 1Zoological Institute/Animal Physiology, University of Tübingen, 72076 Tübingen, Germany, 2Zoological \\ Institute/Developmental Biology, University of Regensburg, 93053 Regensburg, Germany, and ${ }^{3}$ Department of Biology, \\ Brandeis University, Waltham, Massachusetts 02454
}

\begin{abstract}
To study the function of the neuropeptide pigment-dispersing factor (PDF) in the circadian system of Drosophila, we misexpressed the pdf gene from the grasshopper Romalea in the CNS of Drosophila and investigated the effect of this on behavioral rhythmicity. pdf was either ectopically expressed in different numbers of neurons in the brain or the thoracical nervous system or overexpressed in the pacemaker neurons alone. We found severe alterations in the activity and eclosion rhythm of several but not all lines with ectopic pdf expression. Only ectopic pdf expression in neurons that projected into the dorsal central brain severely influenced activity rhythms. Therefore, we conclude that PDF acts as a neuromodulator in the dorsal central brain that is involved in the rhythmic control of behavior. Overexpression of $p d f$ in the pacemaker neurons alone or in the
\end{abstract}

Internal clocks organize the temporal structure of physiological and behavioral functions in probably all organisms. They consist of a circadian pacemaker center that generates an endogenous rhythm of $\sim 24 \mathrm{hr}$, an entrainment pathway for the synchronization with Zeitgebers, and output pathways to effector organs. In recent decades, surprising similarities have been revealed between the pacemaker centers of mammals and insects: both the mammalian suprachiasmatic nucleus (SCN) (Klein et al., 1991) and the insect accessory medulla (for review, see Helfrich-Förster et al., 1998) are located in close vicinity to the optic system. Both pacemaker centers express homologous clock genes that constitute the molecular machinery of the clock (for review, see Dunlap, 1999). Furthermore, both are rich with neuropeptides that are extensively colocalized in the same neurons (Reghunandanan et al., 1993; Petri et al., 1995; Würden and Homberg, 1995). In the $\mathrm{SCN}$, some of these neuropeptides cycle in their abundance

\footnotetext{
Received Nov. 8, 1999; revised Feb. 15, 2000; accepted Feb. 24, 2000.

This work was supported by grants from the Deutsche Forschungsgemeinschaft to C.H.-F. (Fo 207/7-1) and A.H. (Ho 798/5), by a National Institute of Mental Health National Research Service Award to J.H.P., and by a National Institutes of Health grant to Jeffrey C. Hall, Brandeis University. We are grateful for the PDH antiserum from Heiner Dircksen and for the different gal4 lines from Karl-Friedrich Fischbach, Matthew Freeman, Maki Kaneko, and Gerd Technau. We thank Wolf Frommer for the use of the confocal microscope, and Hans Erkert, Wolf Frommer, Jeffrey C. Hall, and Rüdiger Hampp for providing the necessary lab facilities and climate chambers. Furthermore, we thank Peter Pilz for help with the statistics, Gail Fasciani for assistance with the eclosion rhythm tests, and Jeffrey C. Hall and Maki Kaneko for comments on this manuscript.

Correspondence should be addressed to Charlotte Helfrich-Förster, University of Tübingen, Zoological Institute/Animal Physiology, Auf der Morgenstelle 28, 72076 Tübingen, Germany. E-mail: charlotte.foerster@uni-tuebingen.de.

Dr. Park's present address: Department of Biochemistry, Cell and Molecular Biology, University of Tennessee, Knoxville, TN 37996.

Copyright (C) 2000 Society for Neuroscience $0270-6474 / 00 / 203339-15 \$ 15.00 / 0$
}

other neurons that express the clock genes period (per) and timeless (tim) did not disturb the activity rhythm. Such flies still showed a rhythm in PDF accumulation in the central brain terminals. This rhythm was absent in the terminals of neurons that expressed PDF ectopically. Probably, PDF is rhythmically processed, transported, or secreted in neurons expressing per and tim, and additional PDF expression in these cells does not influence this rhythmic process. In neurons lacking per and tim, PDF appears to be continuously processed, leading to a constant PDF secretion at their nerve terminals. This may lead to conflicting signals in the rhythmic output pathway and result in a severely altered rhythmic behavior.

Key words: circadian rhythms; pigment-dispersing factor; neuropeptides; gene misexpression; Drosophila; insects
(Inouye, 1996), and when injected into the SCN they influence cooperatively its spiking activity and provoke phase delays in running activity (Albers et al., 1991, 1992). This suggests that specific peptide interaction is necessary for the regulation of circadian rhythms and that some of these neuropeptides are clock-controlled, as verified recently for the vasopressin gene (Jin et al., 1999).

In insects, the most abundant neuropeptide in the accessory medulla is the "pigment-dispersing factor" (PDF), an ortholog of the crustacean "pigment-dispersing hormone" (PDH) family (Rao and Riehm, 1993). In Drosophila melanogaster, the PDF neurons coexpress the clock genes period ( per) (Helfrich-Förster, 1995), timeless (tim) (Hunter-Ensor et al., 1996; Kaneko et al., 1997), and doubletime (dbt) (Kloss et al., 1998), and they are known as the ventral lateral neurons $\left(\mathrm{LN}_{\mathrm{v}} \mathrm{s}\right)$. The $\mathrm{LN}_{\mathrm{v}} \mathrm{s}$ appear to be critically involved in the circadian control of locomotor activity (Helfrich-Förster, 1998) and are so far the best candidates for individual circadian pacemaker neurons in D. melanogaster.

PDF itself might be involved in the regulation of insect circadian rhythms. Ablation of the PDF neurons in the cockroach caused the rhythm of locomotor activity to disappear (Stengl and Homberg, 1994). This rhythm subsequently reappeared with a time course that matched the extent of regeneration in PDFimmunoreactive neurites (Stengl and Homberg, 1994). Injections of synthetic PDF into the vicinity of the accessory medulla of cockroaches provoked shifts in locomotor activity in a phasedependent manner (Petri and Stengl, 1997). In house flies, PDF is one of the circadian modulators affecting morphology in the optic lobe and compound eye (Pyza and Meinertzhagen, 1996).

To study the function of PDF in the circadian system of 
D. melanogaster we misexpressed the $p d f$ gene of the grasshopper Romalea microptera in the CNS of the fly and investigated the effect of this misexpression on behavioral rhythmicity. We show that ectopic $p d f$ expression in specific neurons led to severe alterations in activity and eclosion rhythms, suggesting that PDF is an integral part of the output pathway of the pacemaker.

\section{MATERIALS AND METHODS}

\section{The upstream activation sequence/GAL4 system}

The upstream activation sequence (UAS)/GAL4 system is a powerful technique for expressing any cloned gene under the control of specific enhancers or promotors (Fischer et al., 1988; Brand and Perrimon, 1993).

A UAS- $p d f$ line was generated using a Romalea cDNA kindly provided by J. Klein (University of Bonn). The cDNA was inserted into the pUAST vector using an EcoRI/XhoI fragment. When the UAS- $p d f$ flies are crossed to flies that carry the gal4 sequence under control of certain enhancers or promotors, GAL4 binds to the UAS, and the transcription of the $p d f$ gene is activated. Consequently, the $p d f$ gene is switched on in all of the cells that express gal4.

We always crossed UAS-pdf males to females of the different gal4 lines in the present experiments.

\section{gal4 lines}

The following gal4 lines were used: Mz1366-gal4, Mz1525-gal4, Mz1172gal4 (gift from K.-F. Fischbach, University of Freiburg, and G. Technau, University of Mainz), elav-gal4 $(\mathrm{P}\{\mathrm{w}[+\mathrm{mW} . \mathrm{hs}]=$ GawB $\}$ elav $[\mathrm{C} 155]$, Bloomington stock center), gmr-gal4 (P\{gmr-gal4\} UF815) (Freeman, 1996), per-gal4 (gift from M. Kaneko, Brandeis University), and pdf-gal4 (Park et al., 2000).

Mz1366-gal4, Mz1525-gal4, and Mz1172-gal4 are enhancer-trap lines that express gal4 in various subsets of CNS cells. In the line elav-gal4, gal4 is under the control of the regulatory sequence of the elav gene and is switched on in all neurons (Robinow and White, 1988, 1991). In gmr-gal4, gal4 expression is controlled by the strong eye-specific enhancer region of the glass gene and present in all photoreceptor cells. In per-gal4, gal4 is controlled by the promotor of the period gene and present in all the cells that normally express per plus some extra cells that do not express native per (Kaneko, 1998). In pdf-gal4, gal4 is fused to the upstream regulatory sequence of the $p d f$ gene (Park et al., 2000) and expressed only in the cells that normally produce PDF.

The level of gal4 expression is generally very robust (Brand and Perrimon, 1993), and GAL4 is known to activate and maintain transcription at high levels (Brand et al., 1994). Furthermore, GAL4 is constitutively present and shows little if any rhythmical variation in its abundance even if under the control of the per promoter. Therefore, $p d f$ misexpression should also occur constitutively and at high levels.

\section{Recording of locomotor activity rhythms}

Locomotor activity of individual flies (males and females) was recorded photoelectrically at $20 \pm 1^{\circ} \mathrm{C}$ as described previously (Helfrich-Förster, 1998). Briefly, we monitored whether a fly was active during a 4 min time span. Activity was scored as 1 ; no activity was scored as 0 . The flies were first monitored for 5-6 d in a $12 \mathrm{hr}$ light/dark cycle (LD 12:12, 1000 lux light intensity) and subsequently for $\sim 15-20 \mathrm{~d}$ under constant darkness (DD). They were then killed so that PDH immunohistology could be performed on their brains (see below).

For each gal4;UAS-pdf line, 20-47 flies were recorded. Flies with the same genetic background but without the UAS-pdf gene served as internal controls for each line.

\section{Estimation of circadian parameters}

At the end of an experiment, the raw data from each recorded fly were transformed to actograms to allow visual judgment of the activity pattern of individual flies.

To compare the activity pattern of the flies under LD, an average day was calculated and plotted as a histogram for each fly. Furthermore, the phase relationship of peak activity to lights on was calculated for each individual fly as described elsewhere (Helfrich-Förster, 2000). The phases of the peaks were given in Zeitgeber time (ZT), whereby the beginning of a 12:12 LD cycle (lights on) is called ZT0 and lights off is called ZT12.

The behavior of the flies under DD was analyzed by periodogram analysis combined with a $\chi^{2}$ test with $5 \%$ significance level (Sokolove and
Bushell, 1978) on the raw unsmoothed data [for detailed description, see Helfrich-Förster (1998)]. A fly was regarded as "simple and robust rhythmic" when visual inspection revealed a rather stable period throughout the recording interval and the periodogram showed a discrete definable peak with a power exceeding $20 \%$ (width of peak $>1 \mathrm{hr}$ ). A fly was classified as "complex rhythmic" when several significant peaks were revealed by periodogram analysis. It was classified as arrhythmic when no rhythmicity could be revealed by visual inspection and no significant period in the circadian range was detected by periodogram analysis or when there were several narrow "spikes" that just reached or barely exceeded the $5 \%$ level (power $<10 \%$, width of peak $<1 \mathrm{hr}$ ).

For each fly daily activity levels were determined throughout the recording time. To compare the overall activity during LD and DD, a mean daily activity level was calculated for each individual fly for the $6 \mathrm{~d}$ in LD and for the first $15 \mathrm{~d}$ in DD. These values were used to calculate means for all fly groups.

\section{Monitoring of eclosion rhythms of elav-gal4;UAS-pdf flies}

elav-gal4 (female) $\times$ UAS-pdf (male) crosses were set up along with elav-gal4 and UAS-pdf parental lines and kept under 12:12 LD conditions at 20 or $25^{\circ} \mathrm{C}$. Flies were transferred to a new bottle every $2-3 \mathrm{~d}$. After the first bottle had seeded for $9 \mathrm{~d}$, adult flies were removed from the last bottles. Pupae were collected and glued to disks that subsequently were placed onto the eclosion monitor as described previously (Konopka et al., 1994). The monitors were placed in 20 or $25^{\circ} \mathrm{C}$ incubators without any external lights (DD). Subsequently, eclosed flies were collected every 30 min and counted automatically. Two separate runs were combined, and the rhythmicity of eclosion was analyzed by $\chi^{2}$ periodogram analysis. Maximum entropy spectral analysis (MESA) was also used to analyze the data because this program was demonstrated to be most powerful in finding weak periodicity (Dowse and Ringo, 1987; Dushay et al., 1990).

\section{Statistics}

The different rhythm parameters of control flies and flies with ectopic $p d f$ expression were compared with an orthogonal two-way ANOVA or $t$ test. Significantly different data sets were then tested for normal distribution by the Kolmogorov-Smirnov test. Periods and evening peaks were not normally distributed only in the case of gmr-gal4;UAS-pdf flies. Therefore, the values of probability ( $p>0.01$ in both cases) were adapted according to Glaser (1978) and regarded as nonsignificant. $\chi^{2}$ analysis (Zar, 1984) was used to reveal whether the distribution of rhythmic, complex rhythmic, and arrhythmic activity patterns was dependent on ectopic $p d f$ expression in the different lines.

\section{Histology}

Experimental animals. After the end of the recording, the flies were transferred from DD to light for 1-2 hr before dissection. The animals were then dissected, and brain whole mounts were immunostained with an antiserum against crab PDH by the peroxidase-anti-peroxidase method exactly as described previously (Helfrich-Förster, 1997). The anti-PDH antiserum (Dircksen et al., 1987) was applied at a dilution of $1: 2000$.

Reconstructions of the PDH-immunoreactive neurons were made with the aid of a Zeiss microscope equipped with a camera lucida attachment.

PDF cycling. To determine whether the amount of PDF in the central brain terminals varies during the LD cycle, flies of the lines elav-gal4; UAS- $p d f, M z 1525$-gal4;UAS- $p d f$, and $p d f$-gal4;UAS- $p d f$ were dissected at two different time points. PDH immunoreactivity was found to be strongest 1-3 hr after lights on and lowest after lights off in the wild type (Park et al., 2000). Therefore, ZT2 and ZT14 were chosen as dissection times. The flies were grown in inverse $\mathrm{LD}$ cycles $(12: 12)$ at $20^{\circ} \mathrm{C}$ with a light intensity of 400-500 lux during the light period. The inversed LD allowed a simultaneous sampling and processing of the flies deriving from the two time points. Exactly the same time intervals were used throughout the immunostaining procedure. At the time of fixation the flies were $15 \mathrm{~d}$ old. All flies were fixed in Zamboni's fixative [4\% paraformaldehyde and $7.5 \%$ picric acid in phosphate buffer $(0.1 \mathrm{M}, \mathrm{pH} 7.4)]$ for $5 \mathrm{hr}$. To facilitate penetration of the aqueous solution, $0.5 \%$ Triton $\mathrm{X}-100$ was added to the fixative. Flies from the dark (ZT14) were handled under a weak red light and kept under these conditions for at least $10 \mathrm{~min}$. After $4 \mathrm{hr}$ fixation the brains were isolated and processed through PDH immunohistochemistry as whole mounts. Anti-PDH was applied for $24 \mathrm{hr}$ at a dilution of 1:2000, then a TRITC-conjugated secondary antibody (Jackson Immunoresearch Laboratories, West Grove, PA; diluted at 1:50) was applied for 4 hr. After rinsing, the brains were embedded in Vectashield embedding 
medium for fluorescence (Vector Laboratories, Burlingame, CA). PDH immunoreactivity was visualized with a fluorescent microscope (LEICA DMR) equipped with a digital camera (Olympus DC10). Several pictures were taken to get all planes of focus of the relevant terminals stained in each brain. These were later mounted in a single picture with the help of Corel PHOTO-PAINT; then the picture was converted into gray scale. The staining intensity of single pixels was scored in gray scale units on the mounted digital pictures using the commercial program DP-soft and DOKU (Olympus). Gray scale units ranged between 0 (black) and 255 (white). The "gray values" of the 10 strongest stained pixels within the terminal region were measured as well as those of 10 corresponding pixels in the background. Mean gray values were calculated for the stained structure and for the background. The difference between both values was regarded as the staining intensity of the terminal region. Staining intensity was determined separately for the corresponding terminals of both brain hemispheres. For each brain, a mean staining intensity was calculated out of the two values.

GFP-staining and double-labeling with anti-PDH. To determine whether PDF was produced in all cells expressing gal4 in the different gal4 lines, the green fluorescent protein (GFP) was used as a reporter for gal4 expression (Brand, 1995) and compared with GAL4-driven $p d f$ expression (see Fig. $3 A-E$ ). Furthermore, the GFP reporter was used to see whether additional $p d f$ was switched on by GAL4 in the PDF cells $\left(\mathrm{LN}_{\mathrm{v}}\right)$ itself. In these cases a double-labeling with anti-PDH was performed (see Fig. $3 F-H$ ). The gal4 lines were crossed to a UAS-gfp line (kindly provided by K.-F. Fischbach, University of Freiburg) carrying the mutant $g f p$ gene $\mathrm{S} 65 \mathrm{~T}$, the protein of which absorbs maximally at $488 \mathrm{~nm}$ (close to filter set FITC). The double-labeling was visualized by a laserscanning confocal microscope (LEICA DMR).

In situ hybridization. To test the mRNA expression of ectopic PDF, the Romalea pdf cDNA was subcloned in pBluscript (P2.4-pBS) and linearized with NotI as a template to transcribe $p d f$ antisense mRNA and linearized with $K p n I$ to transcribe sense $p d f$ mRNA as a negative control. Transcription and labeling were performed by using the DIG-RNA labeling kit from Boehringer Mannheim (Mannheim, Germany). In situ hybridization was performed as described previously (Poeck et al., 1993).

\section{RESULTS}

\section{Misexpression of the pdf gene in virtually all neurons}

In a first attempt we tried to misexpress the $p d f$ gene in all neurons to determine whether such a strong ectopical expression has any effect on the eclosion and the locomotor activity rhythm.

We did this with the help of the elav-gal4 line. The elav gene is expressed in all neurons shortly after their differentiation starts and continues to be expressed (Robinow and White, 1988). It encodes a neural-specific RNA binding protein that is critically involved in neuronal development as well as neuronal maintenance (Yao et al., 1992). Therefore, the ELAV protein is detected in virtually all neurons of the central and peripheral nervous system of embryos, larvae, pupae, and adults, and no difference was found in the ELAV immunoreactivity between 2-d-old brains and 20-d-old adult brains (Robinow and White, 1991). Our studies with the elav-gal4 line in which gal4 expression was visualized with GFP as a reporter were consistent with these findings. Similarly, the $p d f$ gene appeared to be turned on in virtually all neurons when it was under control of the elav promotor as revealed by in situ hybridization with complementary $p d f$ mRNA (data not shown). In contrast to this, the mature peptide revealed by PDH immunohistochemistry was found in many but not in all neurons (see Comparison of GFP expression with PDF expression). PDF was restricted to a well defined subset of neurons in the brain and the thoracic ganglia of L3 larvae $(n=$ $23)$, of late pupae $(n=21)$, of 5- to 10-d-old adult flies $(n=29)$, and of the 25 - to 35-d-old experimental animals $(n=80)$. Generally, more $\mathrm{PDH}$-immunoreactive neurons were found in larvae and pupae than in adults, but not all neurons were stained at any of the developmental stages tested. The PDH-immunoreactive neurons of adult elav-gal4;UAS-pdf flies are shown in Figures 1 and 2 and listed in Table 1. Adult flies showed the same pattern of PDH immunoreactivity regardless of their age, but the overall staining intensity was higher in young flies and seems to decrease with increasing age (Table 1).

\section{PDH immunohistochemistry on adult elav-gal4;UAS-pdf flies}

Ectopic PDF was found in most of the neurons that express the clock genes period and timeless but normally don't contain PDF (Kaneko et al.,. 1997; Kaneko, 1998; Hall, 1998). These were the dorsal lateral neurons (Figs. $1 A, 2 B, L N_{d}$ ) and two groups of dorsal neurons (Figs. $1 C, D N 1, D N 2,2 E, D N$ ). One neuron near the $\mathrm{LN}_{\mathrm{d}}$ group was always strongly labeled and could be traced in its full length. It turned out to be a dorsal giant interneuron (Figs. $1 A, 2 B, D G I$ ) (Ito et al., 1997). PDF was also found in additional neurons close to the $\mathrm{LN}_{\mathrm{v}}$ and $\mathrm{LN}_{\mathrm{d}}$ cluster (Figs. $1 A, 2 B$ ).

Prominent PDF labeling was found in the neuropil of the antennal lobes, in the $\alpha$-, $\beta$-, and $\gamma$-lobes of the mushroom bodies, and in one layer of the central complex (Figs. $1 A, 2 A, C$ ). Furthermore, PDF was reliably revealed in several cell bodies throughout the brain and in processes arising from these. These neurons are described in detail in Table 1 and Figure 1 , and the most conspicuous ones are shown in Figure $2 A-E$.

In the thoracic NS, PDF was present in several superficially located neurons (Fig. $2 F-H$ ). Most of these were located in the third thoracic segment and in the abdominal segments close to the natural PDF-abdominal (PDFAb) neurons (Fig. 2G,H) (cf. Helfrich-Förster and Homberg, 1993). Furthermore, a superficial network of PDH-immunoreactive fibers was stained on the dorsal thoracico-abdominal ganglia and their associated peripheral nerves (Fig. $2 F$ ). This network is similar to the varicose fiber plexus in the neural sheath of the blowfly thoracico-abdominal ganglia that was revealed with anti-serotonin antibodies (Nässel and Elekes, 1985). As in the blowfly, it seems to stem from two pairs of large neurons located in the subesophageal ganglion.

\section{Comparison of elav-gal4-driven GFP and PDF}

The previous experiment showed that $p d f$ gene expression is not necessarily reflected in PDF peptide expression. We made similar observations when we used GFP as a reporter for gal4 expression and compared elav-gal4-driven GFP with elav-gal4-driven PDF. Not all cells that were GFP positive were also PDF positive. No PDF was found in the photoreceptor cells and in the optic lobe, although much GFP was detected in these cells in elav-gal4;UAS$g f p$ flies (Fig. $3 A$ ). Similarly, PDF was not found in the ellipsoid body, although elav-gal4;UAS-gfp flies showed GFP in ring neurons (Fig. 3C). In situ hybridization with complementary $p d f$ mRNA on the retina of gmr-gal4;UAS-pdf revealed massive $p d f$ mRNA in this tissue, although no mature peptide was detected (data not shown). All of these findings indicate that the $p d f$ gene is expressed in all GAL4-containing neurons but that only specific cells provide the functional apparatus to process PDF into its mature peptide form. As a consequence, PDF could be revealed only in a subset of the gal4-expressing cells in the different gal4 lines.

Furthermore, the spatial distribution of GFP and PDF within the neurons was often different. The elav-gal4 line showed strong labeling in the somata of the Kenyon cells of the mushroom bodies when GFP was used as a reporter (Fig. 3D). When crossed to UAS-pdf, no labeling at all was seen in the somata of these cells in adults, but the neurites of the Kenyon cells that constitute the pedunculus, $\alpha-, \beta$-, and $\gamma$-lobes of the mushroom bodies were 


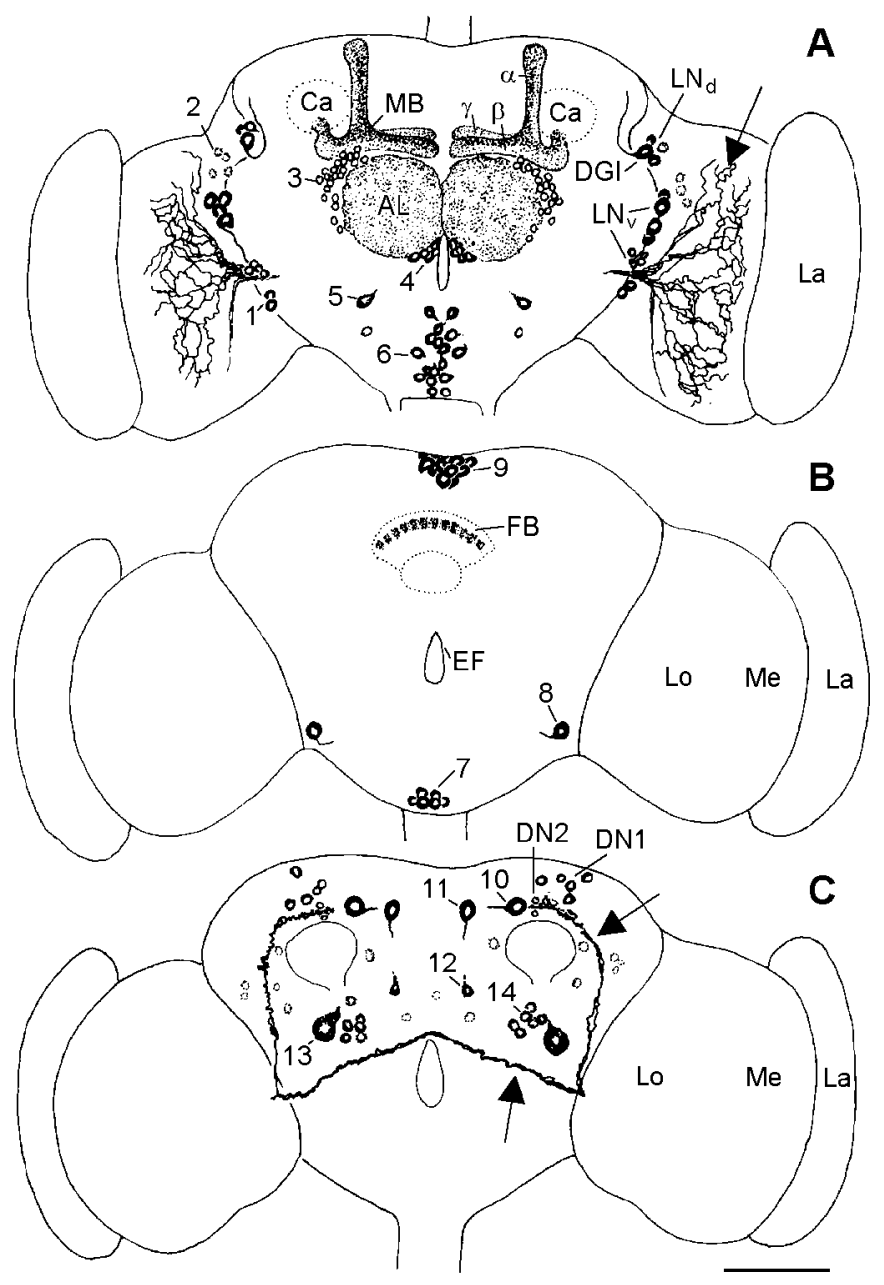

Figure 1. PDH-immunoreactive neurons in the brain of older elav-gal4; UAS- $p d f$ flies. Frontal reconstruction of the anterior $(A)$, medial $(B)$, and posterior $(C)$ brain. The ventral lateral neurons $\left(L N_{\mathrm{v}} \mathrm{s}\right)$ that express natural PDF are shown together with their arborizations (arrows); the varicose network that they form on the surface of the medulla $(\mathrm{Me})$ is only partly shown. The neurons with ectopic PDF are numbered, and only their somata are drawn. The numbers refer to the cell descriptions given in Table 1 and Figure 2. $A$, In the anterior central brain, prominent PDF labeling was found in the neuropil of the antennal lobes $(A L)$ and in the $\alpha-, \beta$-, and $\gamma$-lobes of the mushroom bodies $(M B)$. The fine network of fibers in the glomeruli of the antennal lobes may arise from neurons of cluster 3 and/or cluster 4 . The dense staining in the mushroom bodies belongs to the Kenyon cells that are located near the calyces $(\mathrm{Ca})$. The somata of these cells were stained during a short period in midpupal development but not in adults. One large cell was labeled anteriorly in the tritocerebrum (cluster 5), and many cells were stained in the anterior and medial subesophageal ganglion (cluster 6 ; see also Fig. $2 D$ ). Most of these cells seem to run toward the esophageal foramen. For a detailed description of the other neurons stained in the anterior brain (clusters 1 and 2 and $D G I$ ), see Results and Figure $2 B$. $B$, Medially in the central brain, prominent staining was found in neurons of the pars intercerebralis (cluster 9) and in a layer in the fan-shaped body $(F B)$ of the central complex (see also Fig. 2C). Ventrolaterally in the central brain just at the border to the optic lobe, a large neuron was always strongly stained (cell 8 ). This neuron made connections to its counterpart in the contralateral brain hemisphere and shows conspicuous wide arborizations into the anterior as well as the posterior dorsolateral brain (see Figs. $2 D, 6 A, B$, 10). Neurons of cluster 7 in the ventral medial subesophageal ganglion appear to send fibers through the cervical connective to the thoracic nervous system. $C$, In the posterior brain, many neurons were stained additionally to the per and tim expressing dorsal neurons (DN1 and DN2). Two large conspicuous cells were stained in the pars lateralis. The more dorsolateral-located neuron (cell 10) projected toward the pars intercerebralis and then down the median bundle parallel to the projections of the strongly labeled (Fig. 3E). Similarly, we found strong labeling in a layer of the fan-shaped body of the central complex with UAS-pdf (Fig. 2C) that was almost absent with UAS-gfp. The somata of the cells that give rise to these arborizations (Hanesch et al., 1989) were not labeled with UAS-pdf, but staining was found in such somata with UAS-gfp.

In summary, we observed differences between overall expression of $p d f$ mRNA and the mature peptide PDF, as well as between localization of PDF and GFP within the cell. This suggests that the spatial and temporal expression of the peptide is controlled post-transcriptionally or even post-translationally. PDF appears to be modified, transported, accumulated, and secreted from the neurite independently from $p d f$ gene expression.

\section{Eclosion and locomotor activity rhythms in elav-gal4;UAS-pdf flies}

The analysis of eclosion revealed clear circadian eclosion profiles in the elav-gal4 and UAS-pdf parental lines by visual inspection, periodogram analysis, and MESA (Fig. $4 A, B$ ). In contrast to this, visual inspection of the eclosion profiles of the elav-gal4;UAS-pdf flies showed that eclosion occurred only initially in a rhythmic manner in these flies. Approximately 2-3 d after transfer into $\mathrm{DD}$, elav-gal4;UAS-pdf flies appeared to eclose in an arrhythmic fashion (Fig. $4 C, D$ ). This was similar at 20 and $25^{\circ} \mathrm{C}$. Periodogram analysis and MESA revealed some rhythmicity in the eclosion profiles of these flies at $20^{\circ} \mathrm{C}$ but none at $25^{\circ} \mathrm{C}$. Therefore, we conclude that the rhythm of eclosion is disturbed in elav-gal4; UAS-pdf flies.

For judgement of the locomotor activity rhythm, 87 elav-gal4; UAS-pdf flies (43 males, 44 females) were compared with 71 control flies (33 males, 38 females). Although all controls (Fig. $5 A$ ) were clearly rhythmic, only $44.19 \%$ of the male and $29.54 \%$ of the female elav-gal4;UAS-pdf flies showed such a clear rhythmicity. Most flies were either complex rhythmic or completely arrhythmic (Fig. 8, first two columns). Most of the complex rhythmic flies showed two simultaneously free-running components: one with a period of $\sim 22 \mathrm{hr}$ and one with a period of $\sim 25 \mathrm{hr}$ (Fig. $5 C$ ). Both free-running components seem to arise from the evening peak of activity. The component with the longer period was generally more dominant than that with the short period, and the 32 flies (19 males, 13 females) with clear rhythmicity showed the long period component. The periods of both components were highly significantly different from the periods of the controls (Fig. $9 A$, first two columns).

Under LD conditions, the elav-gal4;UAS-pdf flies extended their activity into the dark period, whereas the activity of controls

\section{$\leftarrow$}

dorsal neurons of cluster 9 (see Fig. $6 C$ showing Mz1172-gal4; UAS-pdf). The adjacent more ventromedial-located second large neuron (cell 11) also projected down the median bundle but additionally arborized in the dorsolateral brain (see Fig. 10). The projections of this neuron were only weakly labeled. A third neuron with small soma was consistently stained ventral to the just described large cells at about the level of the ellipsoid body (cell 12). This cell projected dorsally into the medial dorsolateral brain as well as into the anterior part of the brain where it seems to terminate close to the most dorsal end of the $\alpha$-lobe of the MB. A neuron with huge soma was always stained in the lateral posterior central brain just dorsal to the posterior optic tract (POT) (arrow) (cell 13). Several neurons with smaller somata were clustered around this huge cell (cluster 14). The other stained cells were scattered throughout the posterior brain and could not be recognized individually in different brains. Therefore, these cells were not numbered. $L a$, Lamina; $M e$, medulla; $L o$, lobula complex; $E F$, esophageal foramen; $L N_{\mathrm{d}}$, dorsal lateral neurons; $D G I$, dorsal giant interneuron. Scale bar, $100 \mu \mathrm{m}$. 
Table 1. PDH-immunoreactive neurons in young (2-10 d) and old (20-30 d) flies of the different gal4 lines

\begin{tabular}{|c|c|c|c|c|c|c|c|c|c|c|}
\hline \multirow[b]{2}{*}{ Description of cell clusters } & \multicolumn{2}{|c|}{ elav-gal4 } & \multicolumn{2}{|c|}{ Mz1366-gal4 } & \multicolumn{2}{|c|}{ Mz1525-gal4 } & \multicolumn{2}{|c|}{ Mz1172-gal4 } & \multicolumn{2}{|c|}{ per-gal4 } \\
\hline & $\mathrm{Y}$ & Old & $\mathrm{Y}$ & Old & $\mathrm{Y}$ & Old & $\mathrm{Y}$ & Old & $\mathrm{Y}$ & Old \\
\hline \multicolumn{11}{|l|}{ Anterior brain } \\
\hline $\mathrm{LN}_{\mathrm{v}}$ (large and small) & + & + & - & - & + & + & - & - & + & + \\
\hline $1-3 \mathrm{LN}_{\mathrm{d}}$ & + & + & - & - & $(+)$ & - & - & - & + & $(+)$ \\
\hline DGI & ++ & + & - & - & $(+)$ & - & + & + & ++ & + \\
\hline Two neurons ventral to $\mathrm{LN}_{\mathrm{v}}(1)$ & + & + & + & $(+)$ & - & - & - & - & + & $(+)$ \\
\hline One to four small neurons dorsal to $\mathrm{LN}_{\mathrm{v}}$ (2) & + & $(+)$ & + & - & $(+)$ & - & - & - & - & - \\
\hline$\alpha-, \beta-, \gamma$-lobe of the mushroom body & ++ & ++ & - & - & - & - & - & - & - & - \\
\hline Glomeruli of the AL & ++ & ++ & - & - & ++ & ++ & - & - & - & - \\
\hline Many small neurons dorsolateral to the AL (3) & + & + & - & - & + & + & - & - & - & - \\
\hline $\begin{array}{l}\text { Three to eight small neurons lateral of the EF, } \\
\text { ventromedial to the AL (4) }\end{array}$ & + & + & + & + & ++ & + & - & - & $(+)$ & - \\
\hline One lateral cell in the tritocerebrum (5) & + & + & $(+)$ & - & $(+)$ & - & $(+)$ & - & - & - \\
\hline $\begin{array}{l}\text { Up to } 20 \text { neurons in the anterior medial } \\
\text { subesophageal ganglion (SE) (6) }\end{array}$ & + & + & ++ & + & + & - & + & $(+)$ & + & - \\
\hline \multicolumn{11}{|l|}{ Medial brain } \\
\hline Up to 10 neurons ventrally in the SE (7) & + & + & + & - & + & $(+)$ & + & - & + & - \\
\hline One large neuron in the lateral and ventral brain (8) & ++ & ++ & ++ & ++ & ++ & ++ & - & - & - & - \\
\hline Up to 10 cells in the pars intercerebralis (9) & + & + & ++ & ++ & + & - & ++ & ++ & $(+)$ & - \\
\hline One layer in the fan shaped body & ++ & ++ & + & - & - & - & $(+)$ & - & - & - \\
\hline \multicolumn{11}{|l|}{ Posterior brain } \\
\hline Three to $10 \mathrm{DN} 1$ & + & + & - & - & + & - & + & - & + & + \\
\hline One to two DN2 & $(+)$ & - & - & - & $(+)$ & - & + & - & + & $(+)$ \\
\hline Large neuron in the posterior pars lateralis (PL) close & & & & & & & & & & \\
\hline to the terminals of the small $\mathrm{LN}_{\mathrm{v}}(10)$ & + & + & - & - & - & - & ++ & ++ & + & - \\
\hline Second large neuron in the posterior PL (11) & + & + & ++ & ++ & + & + & - & - & - & - \\
\hline $\begin{array}{l}\text { One neuron at the level of the ellypsoid body that } \\
\text { projects dorsally (12) }\end{array}$ & + & + & - & - & + & + & - & - & - & - \\
\hline $\begin{array}{l}\text { Huge neuron in the lateral posterior brain dorsal to } \\
\text { the POT (13) }\end{array}$ & ++ & + & ++ & + & + & $(+)$ & + & $(+)$ & + & $(+)$ \\
\hline $\begin{array}{l}\text { Three to eight small neurons close to the huge neuron } \\
\text { (14) }\end{array}$ & + & + & ++ & + & + & + & + & $(+)$ & $(+)$ & - \\
\hline $\begin{array}{l}\text { Several small neurons scattered in the posterior brain } \\
\quad \text { (not numbered) }\end{array}$ & $(+)$ & $(+)$ & $(+)$ & - & $(+)$ & - & - & - & - & - \\
\hline \multicolumn{11}{|l|}{ Thoracic nervous system } \\
\hline $\begin{array}{l}\text { Up to four large neurons ventrally in the midline } \\
\text { between segment } 1 \text { and } 2\end{array}$ & + & + & + & - & + & - & + & $(+)$ & - & - \\
\hline $\begin{array}{l}\text { One to four small neurons ventrally in the midline of } \\
\text { segments } 1 \text { and } 3 \text { and between both }\end{array}$ & + & $(+)$ & $(+)$ & $(+)$ & $(+)$ & - & + & + & - & - \\
\hline Large and small cells at the margin of the thoracic NS & + & $(+)$ & + & - & $(+)$ & - & $(+)$ & - & - & - \\
\hline Additional cells in the abdominal segments & + & + & ++ & + & + & - & ++ & ++ & - & - \\
\hline $\begin{array}{l}\text { Horseshoe-shaped cell group located superficially in } \\
\text { the dorsal part of segment } 3\end{array}$ & + & + & + & $(+)$ & $(+)$ & - & ++ & ++ & - & - \\
\hline
\end{tabular}

Y, Young; - , no staining; $(+)$, sometimes weak staining; + , always staining; ++ , always strong staining.

was more restricted to the light period (Fig. 5). The phase determination of morning and evening peak revealed that the elav-gal4;UAS-pdf flies had a highly significant earlier morning peak and later evening peak than the controls (Fig. 9C, first two columns). This was true for both sexes.

Under DD conditions, the elav-gal4;UAS-pdf flies showed a significantly higher activity level than the controls (Figs. 5, 9B, first two columns).

As described previously (Helfrich-Förster, 2000), we found a distinct sexual dimorphism in both fly groups (the elav-gal4;UAS$p d f$ flies and control flies): males showed an earlier morning peak, had a shorter period, and were less active than females (Fig. 9).
Ectopic $p d f$ expression appeared to have qualitatively similar effects on both sexes; however, females reacted somewhat stronger to ectopic PDF than males. They showed a higher amount of arrhythmic flies (Fig. 8) and more extreme period alterations (especially concerning the short period component) than male flies (Fig. 9A). However, the effect of ectopic PDF on the activity level and on the phases of morning and evening peaks was similar in both sexes (Fig. 9B,C).

In summary, misexpression of the $p d f$ gene in the elav-gal4; UAS- $p d f$ flies resulted in severe alterations in eclosion and activity rhythms. The latter were visible as an increase in activity level, an extension of activity into the dark-period, a period increase, 


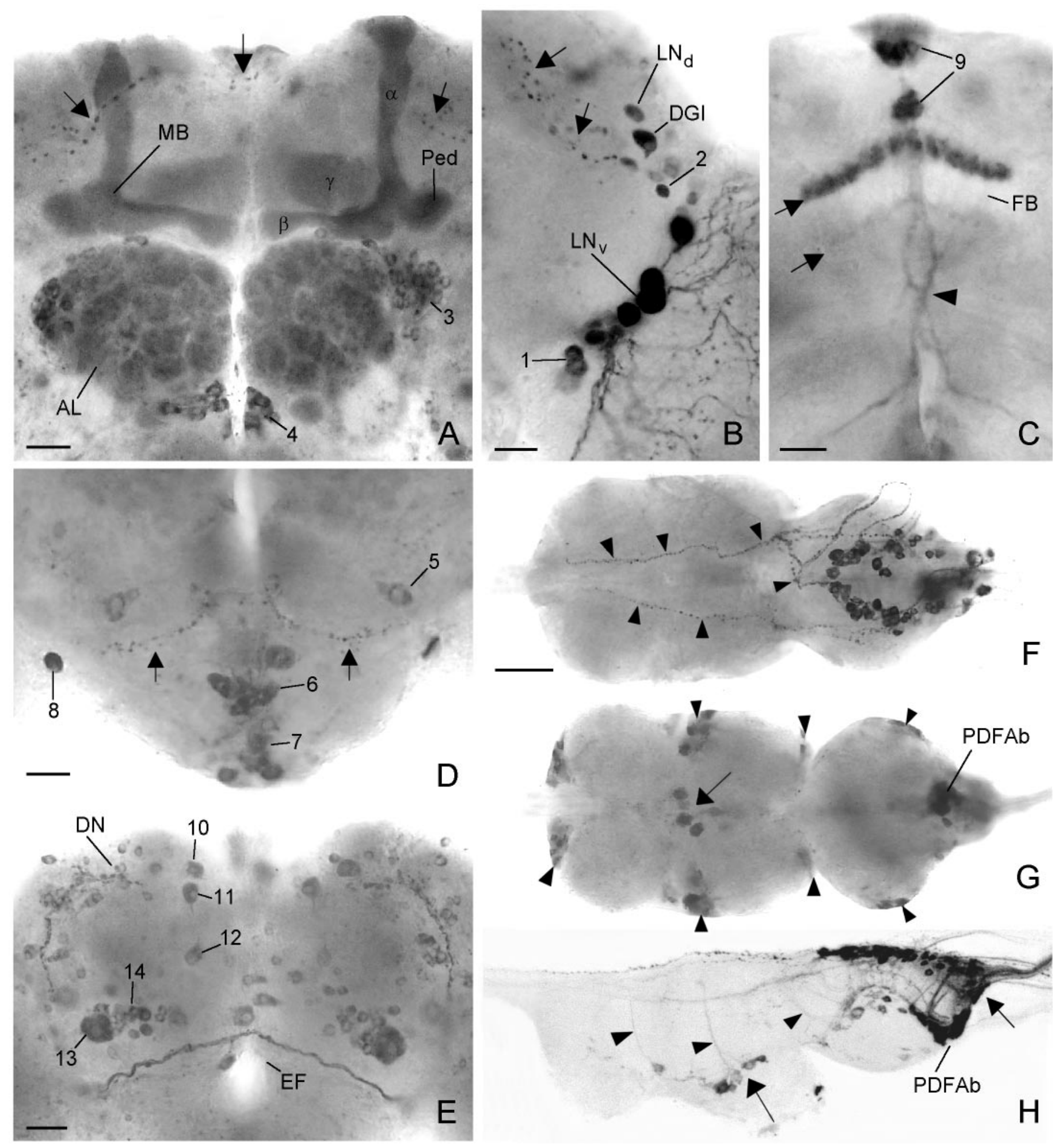

Figure 2. Ectopic PDF in the brain and thoracic nervous system of elav-gal4; UAS-pdf flies revealed by PDH immunocytochemistry. In the anterior central brain $(A)$, strong labeling was found in the $\alpha-, \beta$-, and $\gamma$-lobes and in the pedunculi of the mushroom bodies $(M B)$ and in the glomeruli of the antennal lobes $(A L)$, which were flanked dorsolaterally and ventromedially by two cell clusters (cluster 3 and 4$)$. In the anterior lateral brain $(B)$, the two clusters of lateral neurons $\left(L N_{\mathrm{d}}\right.$ and $\left.L N_{\mathrm{v}}\right)$ expressed PDF $(B)$. One neuron among the $\mathrm{LN}_{\mathrm{d}}$ was a dorsal giant neuron $(D G I)$. Ventrally and dorsally to the $\mathrm{LN}_{\mathrm{v}}$ some additional neurons were stained (cluster 1 and 2). The small arrows in $A$ and $B$ point to projections that arise from the $L N_{\mathrm{d}}$ and form a very dorsal and anterior commissure. In the medial central brain $(C)$, one layer and sometimes an additional faintly labeled second layer were revealed in the fan-shaped body $(F B)$ of the central complex (small arrows). Furthermore, staining was found in neurosecretory cells of the pars intercerebralis (cluster 9). The more ventral cells took their path through the median brain forming a chiasm just above the esophageal foramen (arrowhead). The more dorsal cells projected through the anteriorly located median bundle toward the esophageal foramen (see Fig. $6 C$ ). In the tritocerebrum and subesophageal ganglion $(D)$, several cell clusters were stained (clusters 5, 6, 7, and 8). Most cells of cluster 6 and some of the more ventrally located cluster 7 appeared to project toward the esophageal foramen $(E F)$, whereas two cell pairs of cluster 7 invaded the neural sheath of (Figure legend continues) 

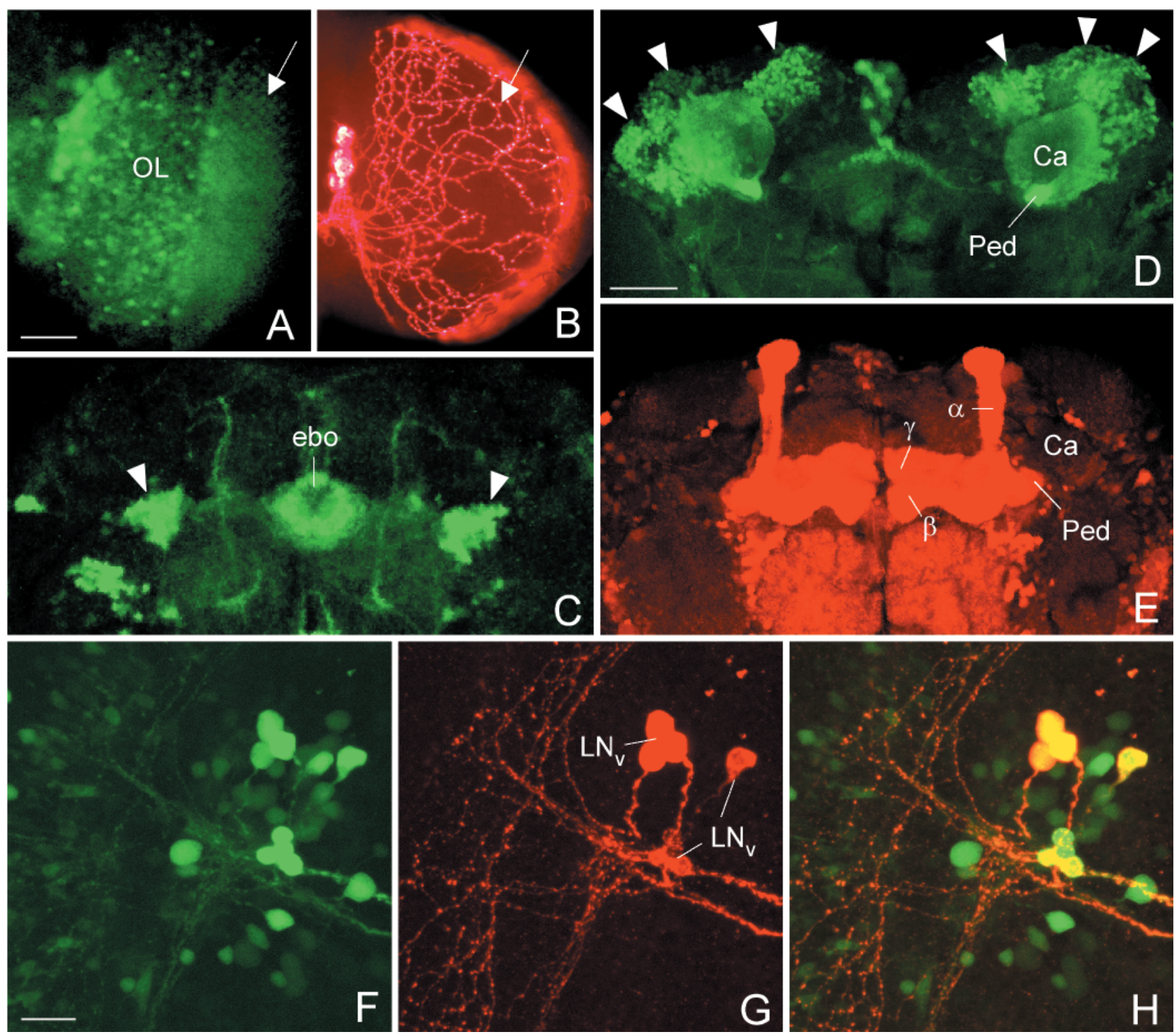

Figure 3. Confocal microscopy to compare GAL4-mediated expression visualized with the reporter GFP and with PDF. The spatial distribution of GAL4-driven GFP and PDF was often different. In the elav-gal4; UAS-gfp line, GFP was prominently revealed in the photoreceptor cells ( $A$, the arrow points to their axonal terminals in the first optic ganglion) and other cells in the optic lobe $(O L)$, whereas no PDF was found in these cells $(B)$. The only PDF labeling present in the optic lobes stemmed from the processes of the $\mathrm{LN}_{\mathrm{v}}$ that formed a varicose network (arrow in $B$ ) on the surface of the medulla. Similarly, no PDF was found in ring neurons of the ellipsoid body (ebo), although these cells strongly expressed GFP (arrowheads in $C$ ). Even the spatial distribution of GFP and PDF in the same neuron was often different: GFP labeling was found in the Kenyon cells $(D$, arrowheads) and their corresponding dendrites in the Calyx $(\mathrm{Ca})$ of the mushroom body. However, ectopic PDF was only revealed in the $\alpha-, \beta$-, and $\gamma$-lobes and the pedunculus (Ped) of the mushroom bodies $(E)$. To test whether GAL4-mediated expression was present in the $\mathrm{LN}_{\mathrm{v}} \mathrm{s}$, double-labeling with anti-PDH was performed on the relevant gal4-lines whereby gal4 expression was visualized with GFP. In the line Mz1525-gal4; UAS-gfp, GAL4-mediated GFP $(F)$ was found in all PDF-labeled LN $\mathrm{v}_{\mathrm{v}}$ $(G)$ as revealed by superposition of GFP and PDF labeling $(H)$. Scale bars (shown in $A$ ) $A, B, 50 \mu \mathrm{m}$; (shown in $D$ ) $C, D, E$, $50 \mu$ m; (shown in $F$ ) $F-H$, $20 \mu \mathrm{m}$.

the cervical connective and that of the thoracic NS (arrowheads in $F$ ). The cell pair number 8 appeared to be contralaterally connected (arrows) and formed conspicuous wide arborizations in the anterior and dorsolateral brain that are shown in Figure $6, A$ and $B$. In the posterior central brain $(E)$, a large number of cells with ectopic PDF were found; only some of these were numbered (clusters 10-14). In the third thoracic neuromere of dorsal thoracic NS $(F)$, ectopic PDF was revealed in many superficially located cells that were arranged in a horseshoe-shaped manner. Whether the varicose network of fibers on the surface of the thoracic NS (arrowheads) stems partly from these cells or entirely from those located in the subesophageal ganglion of the brain (cluster 7) is unclear. In the ventral thoracic NS $(G)$, several large cells were stained in the midline of the second thoracic neuromere (large arrow in $G$ and $H$ ) plus several smaller ones were stained at the borders between the three neuromeres (arrowheads). In the abdominal neuromeres, many cells (right arrow in $H$ ) were stained in addition to the natural PDFAb cells. A sagittal view of the thoracic NS $(H)$ shows that many of the ventrally located cells projected dorsally (arrowheads). Scale bars: $A-E, 20 \mu \mathrm{m}$; (shown in $F$ ) $F-H, 100 \mu \mathrm{m}$. 


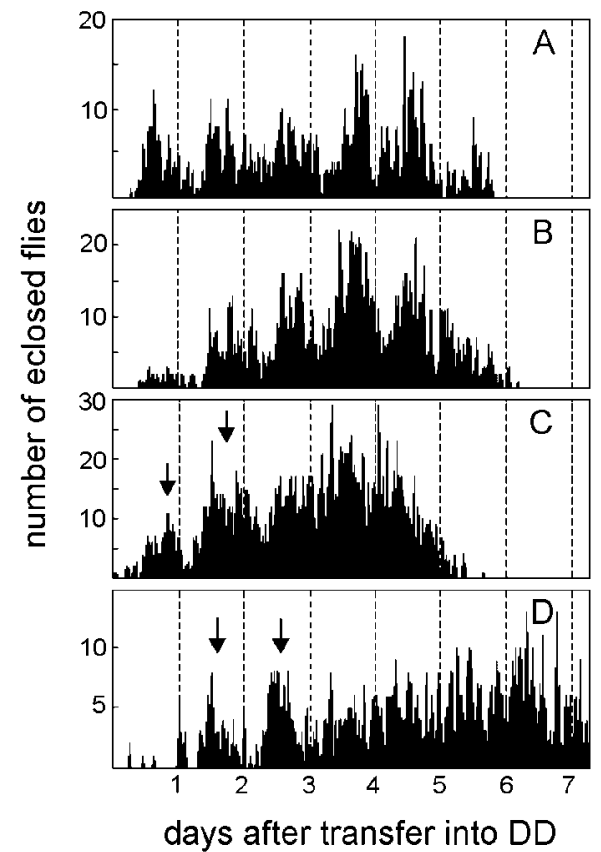

Figure 4. Eclosion rhythm in UAS-pdf flies $(A)$, elav-gal4 flies $(B)$, and elav-gal4; UAS-pdf flies $(C, D)$. The parental lines (UAS-pdf and elav-gal4 flies) were recorded at $25^{\circ} \mathrm{C}$. The elav-gal4; UAS-pdf cross was monitored at $25^{\circ} \mathrm{C}(C)$ and at $20^{\circ} \mathrm{C}(D)$. At both temperatures only two clear eclosion peaks (arrows) could be seen by visual inspection in these flies; then eclosion slowly became arrhythmic. In the parental lines, eclosion remained clearly rhythmic throughout the $6 \mathrm{~d}$ of monitoring. Periodogram analysis and MESA revealed strong rhythmicity in the parental lines and some rhythmicity in elav-gal4; UAS-pdf flies at $20^{\circ} \mathrm{C}(D)$, but no rhythmicity at $25^{\circ} \mathrm{C}(C)$.

and a destabilization of the free-running rhythm becoming evident in the frequent occurrence of complex rhythmic or arrhythmic activity patterns. Therefore, we conclude that PDF strongly affects behavioral rhythms.

\section{Misexpression of PDF in specific groups of neurons}

To reveal what neurons with ectopic PDF are responsible for the alterations in the activity rhythm, we misexpressed PDF only in subgroups of the PDF-positive cells of the elav-gal4;UAS-pdf flies (Table 1, Fig. 6). Four different gal4 lines were used for this purpose: Mz1366-gal4;UAS-pdf, Mz1525-gal4;UAS-pdf, Mz1172gal4;UAS-pdf, and gmr-gal4;UAS-pdf. In gmr-gal4;UAS-pdf, the $p d f$ gene was expressed in all photoreceptor cells. Nevertheless, we did not find any mature PDF in these cells by PDH immunohistochemistry, and the flies showed a normal locomotor activity rhythm (Figs. 8, 9). In the other gal4 lines, PDF was restricted to specific subsets of neurons in the brain and thoracic NS (Table 1). Generally, more PDF cells were detected in pupae and younger flies than in old ones. Furthermore, the intensity of PDH immunoreactivity decreased with increasing age of the flies. This was most evident in the thoracic NS of the lines Mz1366-gal4 and Mz1525-gal4 (Table 1). Especially in older Mz1525-gal4;UAS-pdf flies, PDF was virtually absent from the thoracic NS. Similarly, PDF was restricted to very few brain neurons in older flies of this line. Strong PDF labeling was found only in neurons 8, 11, and 12 (Fig. 6A,B) and in the $\mathrm{LN}_{\mathrm{v}}$ (Fig. $3 F-H$ ). Nevertheless, $M z 1366$ gal4;UAS-pdf and Mz1525-gal4;UAS-pdf flies exhibited a severely altered locomotor activity rhythm that was very similar to elavgal4;UAS-pdf flies (Figs. 7-9). The majority of flies were either
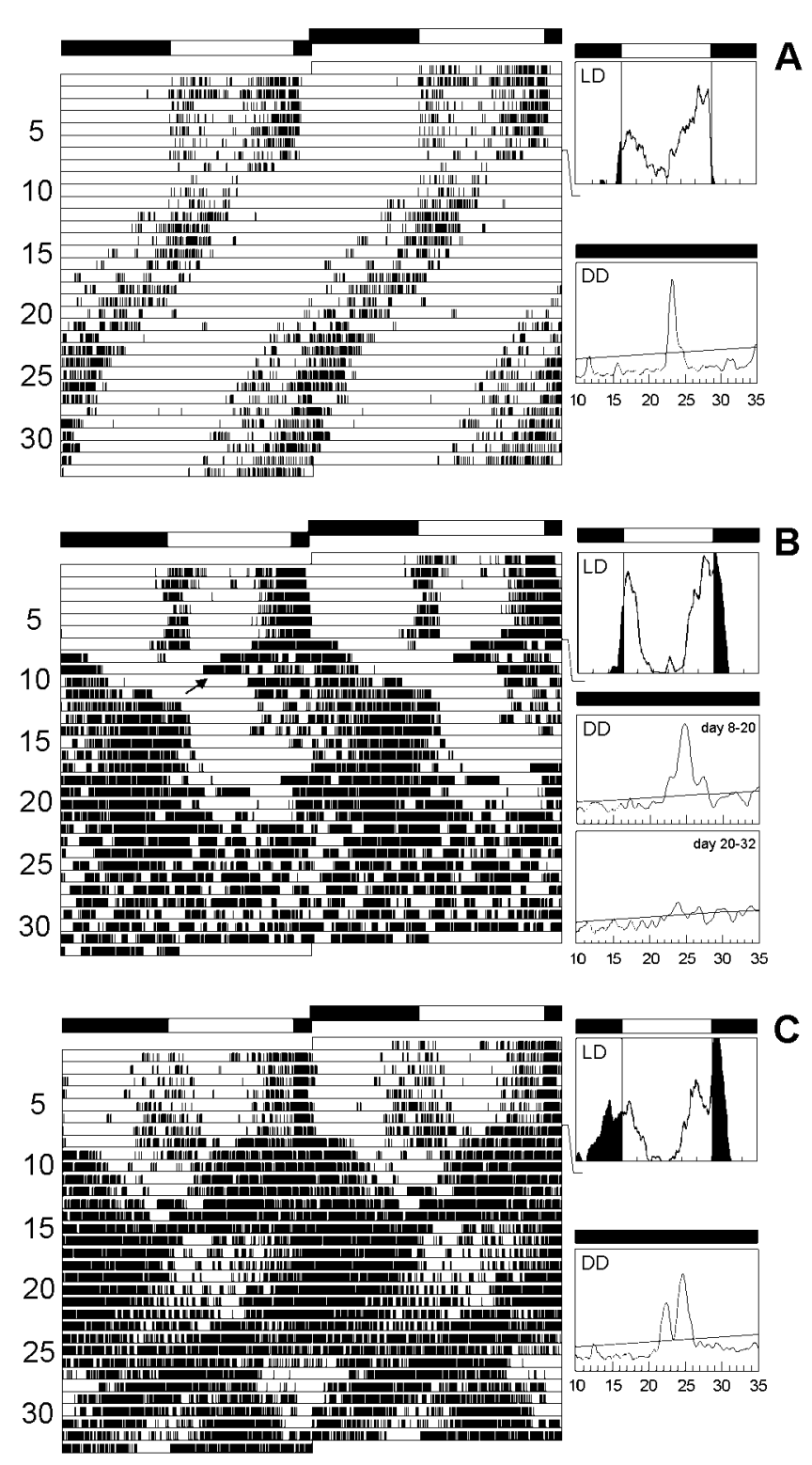

Figure 5. Locomotor activity rhythms in elav-gal4; UAS-pdf and control flies. All flies were first recorded for $7 \mathrm{~d}$ under a $12 \mathrm{hr}$ light/dark cycle $(L D)$ and subsequently for $26 \mathrm{~d}$ under continuous dark conditions $(D D)$. For every fly actogram, average days during LD and periodograms of the free-running rhythm in DD are shown. The first panels $(A)$ illustrate the rhythmic behavior of a typical male control (wild-type) fly. During LD, the fly's activity pattern was clearly bimodal: a morning and an evening peak of activity could be distinguished in the average day. Activity starts $\sim 0.5 \mathrm{hr}$ before lights on, but most activity was restricted to the light phase. During DD the fly was clearly rhythmic and showed a more or less stable, free-running period. A slight lengthening in period occurred from day 20 onward. Such smaller period changes were frequently observed in wildtype flies and were not considered further in this study. $B$ and $C$ show the activity pattern of two typical male elav-gal4; UAS-pdf flies. During LD both flies had a bimodal activity pattern similar to the wild type, but their activity was less restricted to the light phase. Especially the fly in $C$ showed a considerable amount of activity during the dark phase. During $\mathrm{DD}$, both flies had a complex activity pattern. The fly in $B$ showed a main free-running component with a long period, but shortly after transfer into DD a second free-running component with a short period appears to arise from this main component (arrow). Both components clearly originate from the evening peak of activity. From day 22 onward, the fly became arrhythmic (lower periodogram). The fly in $C$ showed two simultaneously free-running components throughout the recording time in DD. 

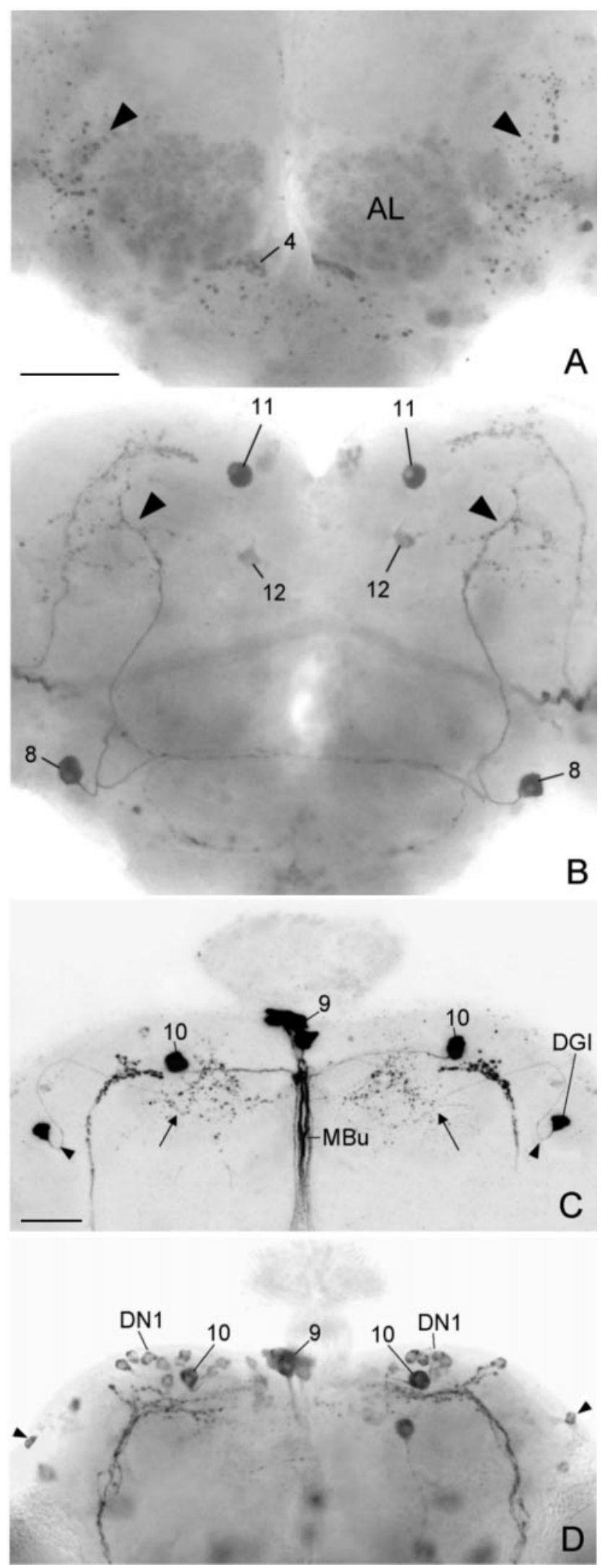

Figure 6. Ectopic PDF in the central brains of three different gal4; UAS- $p d f$ lines that express PDF only in subsets of the neurons that were found in the elav-gal4; UAS-pdf line. A typical Mz1525-gal4; UAS-pdf brain is depicted from an anterior $(A)$ and posterior $(B)$ plane of focus. This brain stemmed from the experimental animal with arrhythmic ac- arrhythmic or showed complex rhythmicity that was composed of two activity components, the activity level of the flies under DD was increased, and the phase of the morning peak was advanced.

Some minor differences were observed in the overall activity pattern between these two lines and those of the line elav-gal4; UAS- $p d f$ : in Mz1366-gal4;UAS-pdf flies with complex rhythmicity, the components with short and long periods were of equal strength, and in Mz1525-gal4;UAS-pdf flies, the short period component was the more prominent one. (In the first line, three of the five clearly rhythmic flies had a long period; the remaining two had short periods. In the latter line, six of such flies had short periods, and only two had long ones.) Furthermore, the effect of PDF on the phase of the evening peak was different in males and females of Mz1366-gal4;UAS-pdf and Mz1525-gal4;UAS-pdf flies: ectopic PDF induced a phase delay in the evening peak only in female flies (Fig. 9C). This interaction between sex and evening peak was significant in both lines, whereas we did not observe such an effect in the line elav-gal4;UAS-pdf (Fig. 9C).

Despite these minor differences among Mz1366-gal4;UAS-pdf, $M z 1525$-gal4;UAS-pdf, and elav-gal4;UAS-pdf flies, the most important observation is that all three lines exhibited a severely altered activity rhythm, suggesting that the latter is attributable to $p d f$ misexpression in only a few neurons common to all three lines (see below).

Adult flies of line Mz1172-gal4;UAS-pdf showed an almost complementary PDF expression pattern to the two lines that were just described. Older flies of this line revealed strong PDF labeling in the thoracic NS, in neurons 9 and 10, and in the DGI (Table 1, Fig. $6 C$ ). These flies showed a normal rhythmic behavior; we did not observe an increase in the number of complex and arrhythmic flies (Fig. 8). Nevertheless, the period was slightly shortened and the phase of the morning peak a bit advanced, as compared with the controls (Fig. 9A,C). These effects were much less dramatic, however, than those observed in the previous lines. Therefore, we conclude that misexpression of PDF in the thoracic NS and the neurosecretory cells of the pars intercerebralis (plus neuron 10 and the DGI) has little influence on the rhythm of locomotor activity and does not disturb general rhythmicity at all.

Interestingly, larvae and pupae of Mz1172-gal4;UAS-pdf showed strong PDH immunoreactivity in many more neurons than did adults. Such ectopic PDF during development obviously had no severe effect on adult rhythmicity.

tivity pattern shown in Figure $7 C$. Ectopic PDF was found in only a few neuron pairs in this brain. The most prominent one was neuron 8 , which showed arborizations in the anterior brain (arrowheads in $A$ ) as well as in the dorsolateral posterior brain (arrowheads in $B$ ). The somata of neuron pair 11 were also strongly stained, but their arborizations into the dorsolateral brain and down the median bundle (see Fig. 10 $A$ ) were not revealed. Weak staining was found in the soma of neuron 12 and in neurons of cluster 4 plus their putative arborizations in the antennal lobes $(A L)$. In the line $M z 1172$-gal4; UAS-pdf $(C)$, neuron pair 10 was prominently revealed together with neurons in the pars intercerebralis (cluster 9). All of these cells projected down the median bundle $(M B u)$. The other arborizations (arrow) in this brain stemmed from the dorsal giant interneuron (DGI). The arrowheads point to the characteristic bend of the neurite close to its soma (cf. Ito et al., 1997). In the per-gal4; UAS-pdf line $(D)$, the first group of dorsal neurons (DN1) was clearly stained. Neuron 10 was located among these cells; whether it belongs to the DN1 is unclear. The DN2 neurons were not revealed in this particular brain, but some DN3 appear to be stained (arrowhead). PDF was also found in some neurons that naturally do not express per and tim as in the neurons of cluster 9 [compare Table 1 and Kaneko (1998)]. Scale bars: (shown in $A$ ) $A, B, 50 \mu \mathrm{m}$; (shown in $C$ ) $C, D, 50 \mu \mathrm{m}$. 

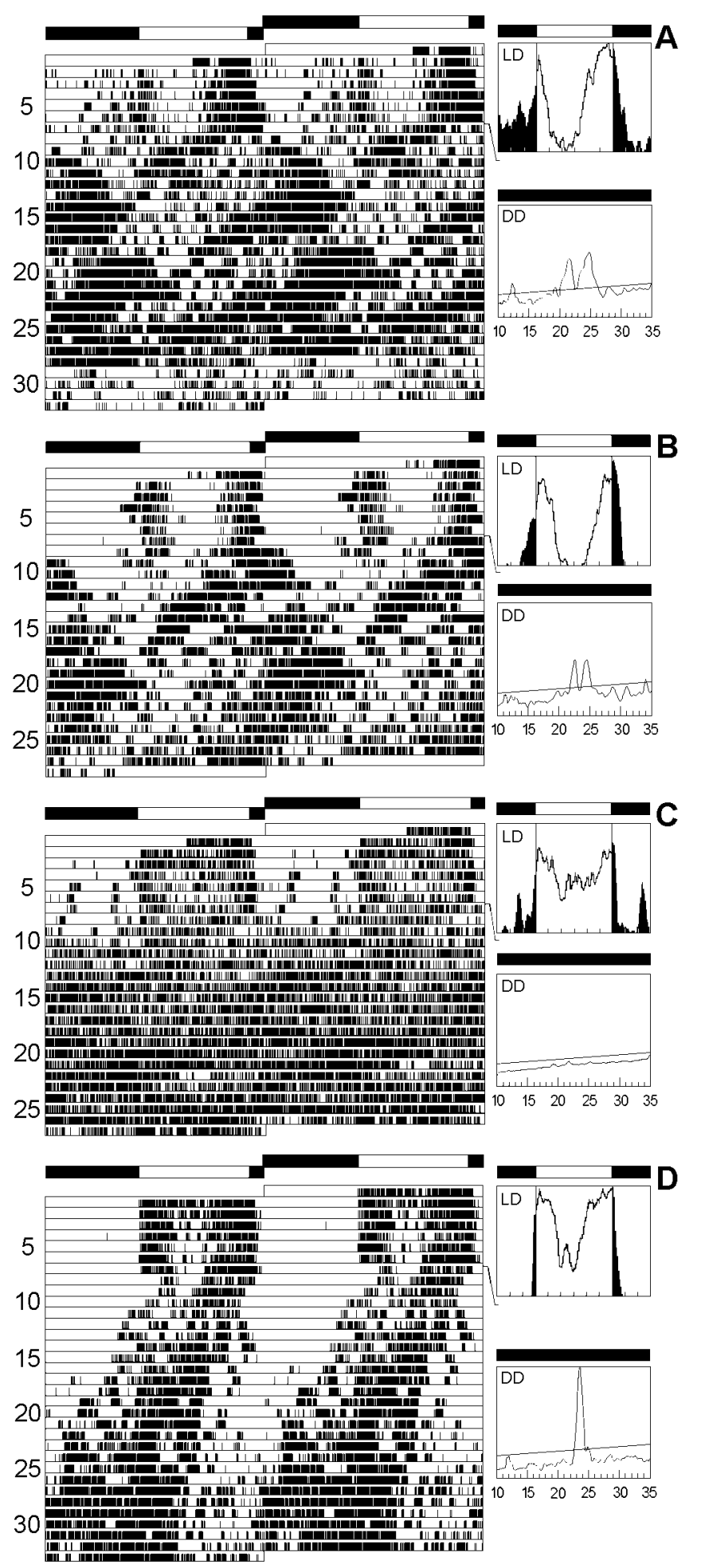

Figure 7. Locomotor activity rhythms in three different gal4; UAS-pdf lines. The recording schedule is as in Figure 5. The Mz1366-gal4; UAS-pdf line $(A)$ and the $M z 1525$-gal4; UAS-pdf line $(B, C)$ showed a behavior that was very similar to that of the elav-gal4; UAS-pdf line (Fig. $5 B, C$ ), although fewer neurons showed ectopic PDF expression. The flies were either complex rhythmic $(A, B)$ or arrhythmic $(C)$. The brain of the arrhythmic Mz1525-gal4; UAS-pdf fly $(C)$ is depicted in Figure 6, $A$ and $B$. When PDF was overexpressed in the $\mathrm{LN}_{\mathrm{v}} \mathrm{s}$ only ( $p d f$-gal4; UAS-pdf fly), locomotor activity rhythm was wild-type-like $(D)$.

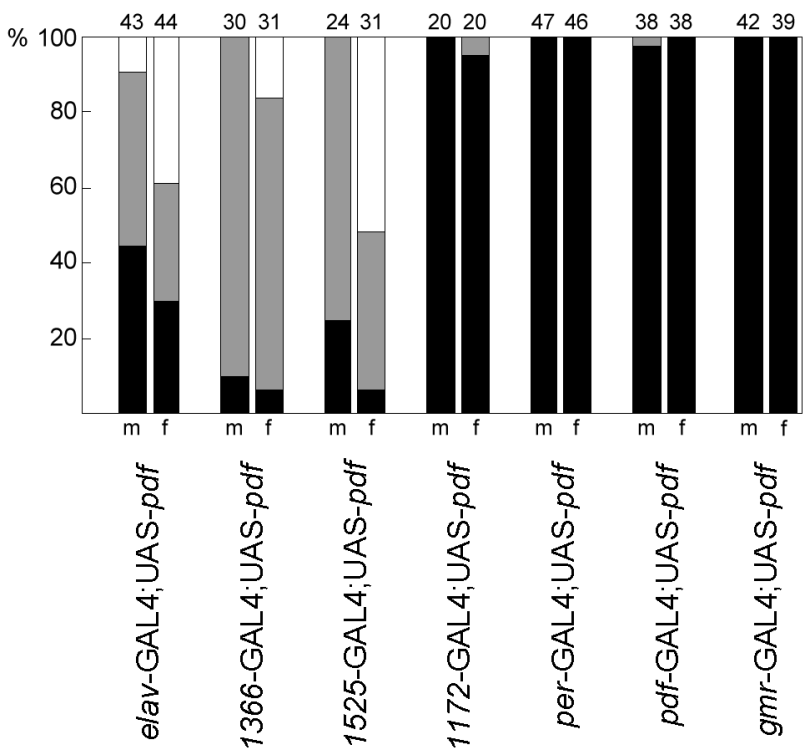

Figure 8. Distribution of locomotor activity patterns in the different lines with ectopic PDF. The percentages of normal rhythmic flies (black bar), complex rhythmic flies (gray bar), and arrhythmic flies (white bar) are shown for males $(m)$ and females $(f)$ of the different groups. Numbers of tested animals are given on top of the bars. Most control flies (95-100\%) were normally rhythmic; therefore, these were not included in the present graph. Data of controls and of the corresponding flies with ectopic PDF were arranged in contingency tables for $\chi^{2}$ analysis (males and females pooled; complex rhythmic and arrhythmic flies pooled). Rhythmicity was strongly dependent on ectopic PDF in the first three lines $(p<0.0001$, $\left.\chi^{2}>63.30\right)$ but not in the remaining four lines $\left(p>0.9160, \chi^{2}<0.008\right)$. In the lines elav-gal4; UAS-pdf and Mz1525-gal4; UAS-pdf, the distribution of rhythmic, complex rhythmic flies was dependent on the sex $(p<$ $\left.0.006, \chi^{2}>10.20\right)$

\section{Overexpression of PDF in the LN and DN}

Two of the three lines with altered activity rhythm (elav-gal4; UAS- $p d f$ and Mz1525-gal4;UAS- $p d f$ ) misexpressed the $p d f$ gene in the $\mathrm{LN}_{\mathrm{v}}$ in addition to the ectopic expression in other neurons that was described above (Fig. $3 G, H$ ). The $\mathrm{LN}_{\mathrm{v}} \mathrm{s}$ are the putative circadian pacemaker neurons of Drosophila, and overexpression of the $p d f$ gene in these cells alone may influence locomotor activity and be responsible for the altered rhythmicity in these two lines. To test this, we overexpressed the $p d f$ gene in the $\mathrm{LN}_{\mathrm{v}}$ alone ( $p d f$-gal4;UAS- $p d f$ line) or in the $\mathrm{LN}_{\mathrm{v}}, \mathrm{LN}_{\mathrm{d}}$, and $\mathrm{DN}$ (per-gal4;UAS-pdf line) (Fig. 6D).

In both lines we did not see any effect on general rhythmicity (Figs. 7D, 8), but as in the line Mz1172-gal4;UAS-pdf, we observed a slight period shortening and a minor advance in the morning peak (Fig. 9A,C). The period shortening was only significant, however, in the $p d f$-gal4;UAS-pdf line (Fig. 9A), whereas the advance of the morning peak was only significant in the per-gal4; UAS-pdf line (Fig. 9D). Furthermore, both lines showed a lower activity level than the controls (Fig. 9B). In per-gal4;UAS-pdf flies, this was true in LD and DD, whereas in $p d f$-gal4;UAS-pdf flies, activity was reduced only under LD conditions. These small effects on rhythmic parameters can be attributed to $p d f$ overexpression in the $\mathrm{LN}_{\mathrm{v}}$ as well as to $p d f$ misexpression in the $\mathrm{LN}_{\mathrm{d}}$ and DN. Probably both contribute because the observed effects appeared stronger in per-gal4;UAS-pdf flies than in $p d f$-gal4;UAS$p d f$ flies. These results also suggest that the $p d f$ gene is indeed overexpressed in the $\mathrm{LN}_{\mathrm{v}}$ in both lines (although this is hard to confirm by $\mathrm{PDH}$ immunohistochemistry) but that the severe 


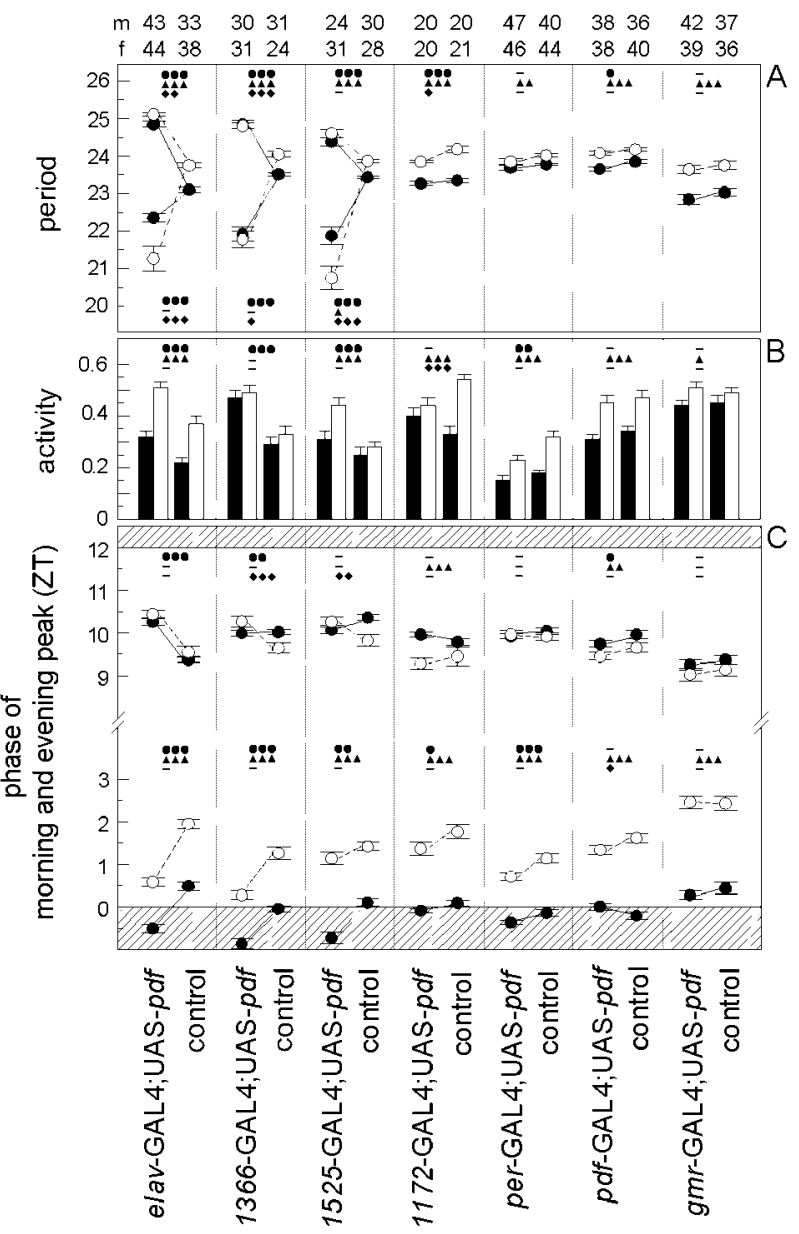

Figure 9. The effects of ectopic PDF on locomotor activity. Depending on its expression pattern in the different gal4 lines, PDF affected the free-running period $(A)$, the daily activity level under DD conditions $(B)$, and the phases of morning and evening peaks under LD conditions $(C)$. Phases of morning and evening peaks are given in Zeitgeber time $(Z T)$, whereby lights on is ZT0 and lights off is ZT12 $(C)$. The shaded areas in $C$ indicate the beginning and end of the dark period of the LD cycle, respectively. In many cases the morning peak occurred during darkness before lights on. Mean values $( \pm \mathrm{SE})$ of the different parameters are shown for the flies with ectopic PDF and their corresponding internal controls of each gal4 line (see description at bottom of Figure). Closed circles and black bars represent mean values for males, and open circles and white bars represent such values for females. Numbers of tested animals are given on top of the bars for males $(m)$ and females $(f)$ separately. (For period determination the numbers of analyzed flies were in some cases lower than the numbers given at the top, because the periods of arrhythmic flies could not be determined. In the case of complex rhythmicity with two simultaneously free-running components, the mean periods of both components are shown in the graph.) The data of controls and experimental animals of each line were compared with an orthogonal two-way ANOVA to reveal the influences of PDF misexpression $(\boldsymbol{\bullet})$, of sex $(\boldsymbol{\Delta})$, and of interactions of both $(\bullet)$ on the different parameters. Three symbols represent a probability for a significant effect of $p<0.001$ $\left(F_{(1, \mathrm{df})}>12.30, \mathrm{df}=77-173\right)$; two symbols represent a probability of $p<$ $0.01\left(F_{(1, \mathrm{df})}>7.30, \mathrm{df}=77-173\right)$; one symbol represents a probability of $p<0.05\left(F_{(1, \mathrm{df})}>3.90, \mathrm{df}=77-148\right)$; and a minus marks the values that were not significantly different $\left(p>0.05 ; F_{(1, \mathrm{df})}<3.84\right.$, df $\left.=87-173\right)$. In case of complex rhythmicity with two simultaneously free-running components, the periods of both components were compared with the single periods of the controls $(A)$. PDF misexpression had significant effects on period $(A)$, activity level $(B)$, and phases of morning and evening peak (C) mainly in the first three gal4 lines. In the other four lines, PDF had only mild effects on some of the rhythmic parameters. A sexual dimorphism was found in all lines. Female flies had significantly longer periods, a higher activity level (except for Mz1366-gal4; UAS-pdf flies and con- alterations in locomotor activity rhythms observed in elav-gal4; UAS-pdf and Mz1525-gal4;UAS-pdf flies are not caused by $p d f$ overexpression in the pacemaker neurons.

To further increase the transcription rate of $p d f$, we took advantage of the temperature sensitivity of GAL4-mediated ectopic expression, which is stronger at $29^{\circ} \mathrm{C}$ than at 25 or $18^{\circ} \mathrm{C}$ (Brand et al., 1994). Therefore, we recorded 20 per-gal4;UAS-pdf flies additionally at $29^{\circ} \mathrm{C}$. Indeed, $\mathrm{PDH}$ immunohistochemisty revealed a subjectively stronger staining intensity in the somata of the LNs and DNs at this temperature. Nevertheless, general rhythmicity was not affected in these flies. Therefore, we conclude that overexpression of the $p d f$ gene in the pacemaker cells themselves or in the related DNs does not disturb the activity rhythm and that the severely altered rhythmicity observed in the lines elav-gal4;UAS-pdf, Mz1525-gal4;UAS-pdf, and Mz1366-gal4;UAS$p d f$ must be caused by ectopic PDF.

\section{Where is the site of action of ectopic PDF?}

When comparing the pattern of ectopic PDF in the different gal4 lines (Table 1), neurons 8 and 11 were the only cells that showed ectopic PDF expression in all lines with abnormal rhythmicity but not in the ones with normal rhythms. Therefore, ectopic PDF in these two neurons is most likely the cause for the abnormal activity rhythms. Both neurons had extensive arborizations in the dorsolateral brain close to the terminals of the $\mathrm{LN}_{\mathrm{v}}$ (Fig. 10A), a region that is crucial for the transfer of circadian signals (Helfrich-Förster, 1998). In the wild type, PDF accumulates rhythmically in the $\mathrm{LN}_{\mathrm{v}}$ terminals, showing a maximum at ZT0-3 and a minimum at ZT12-15 (Park et al., 2000). This rhythm is absent in the arrhythmic mutants per $^{0}$ and $\operatorname{tim}^{\circ}$, and in the short period mutant per $^{\mathrm{s}}$ it continues with a period of $20 \mathrm{hr}$ under constant dark conditions (DD), suggesting that it is dependent on per and tim. If we assume that neurons 8 and 11, which don't express the per and tim genes, continuously release PDF into the dorsolateral brain, it is reasonable that this will influence the interneurons downstream of the pacemaker cells. However, it is hard to understand why overexpression of the $p d f$ gene in the pacemaker cells itself does not affect rhythmicity at all. A possible explanation would be that PDF is rhythmically accumulated and released from the $\mathrm{LN}_{\mathrm{v}}$ terminals despite its gene overexpression. To test this we looked at PDF labeling in the terminals at the time of maximal (ZT2) and minimal PDF staining (ZT14). In both the pdf-gal4;UAS-pdf line and the Mz1525-gal4;UAS-pdf line, PDF staining in the $\mathrm{LN}_{\mathrm{v}}$ terminals was significantly stronger at ZT2 than at ZT14 (Fig. 10B). In contrast to that, PDF staining intensity at the terminals of neuron 8 in Mz1525-gal4;UAS-pdf flies was similar at both time points (Fig. 10B). This indicates that PDF accumulation and release occurs rhythmically in the pacemaker neurons and that this rhythm is not altered by overexpression of the $p d f$ gene in these cells. In neuron 8, which does not express the clock genes period and timeless, no such rhythm in

\footnotetext{
trols), and a later morning peak than males. In the lines Mz1172-gal4; UAS- $p d f$ and $p d f$-gal4; UAS- $p d f$ plus controls, we also found an effect of sex on the evening peaks: males had a later evening peak than females $(C)$. Different effects of PDF in both sexes (interaction of PDF and sex) were mainly found in period lengthening or shortening $(A)$ and on the phase of the evening peak $(C)$ in the first three lines. Furthermore, such effects were present in period shortening and activity level changes in the line Mz1172-gal4; UAS-pdf, and on the phase of the morning peak in the line $p d f$-gal4; UAS-pdf. For other details see Results.
} 

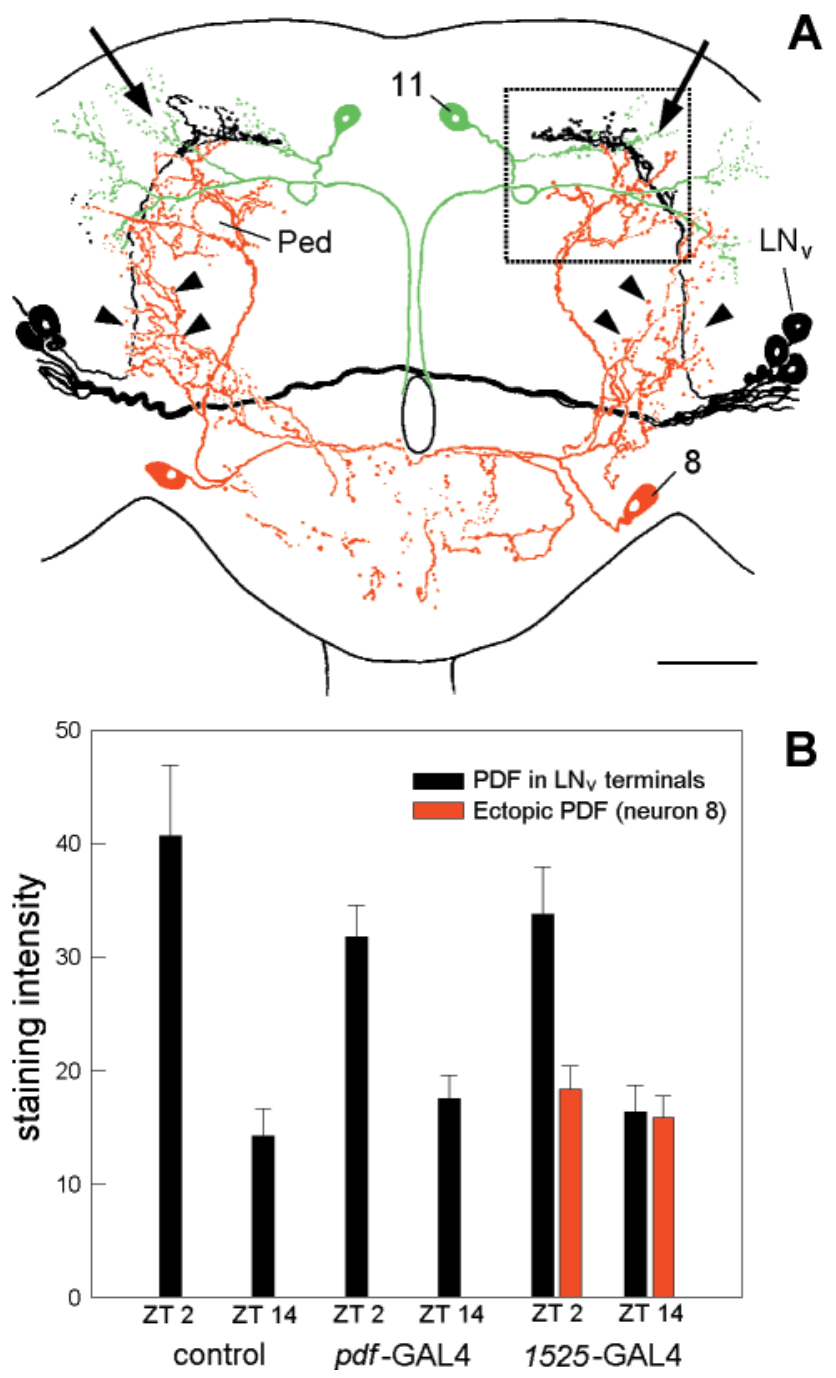

Figure 10. Arborization pattern of the $\mathrm{LN}_{\mathrm{v}}$ (black), neuron 8 (red), and neuron 11 (green) in the central brain of D. melanogaster $(A)$, and PDF-staining intensity in the central brain terminals of the $\mathrm{LN}_{\mathrm{v}}$ in the wild-type and the lines $p d f$-gal4; UAS-pdf and Mz1525-gal4; UAS-pdf (B) and of neuron 8 (in Mz1525-gal4; UAS-pdf). All three neurons show a partial overlap in their arborization fields in the dorsolateral protocerebrum $(A$, arrows $)$. Neuron 8 had additional arborizations in the medial and ventral anterior brain (arrowheads). Ped, Pedunculus of the mushroom body; $L N_{\mathrm{v}}$, ventral lateral neuron. The rectangle indicates the terminal area that was used for judgement of staining intensity. Scale bar, $50 \mu \mathrm{m}$. Staining intensity $(B)$ was judged at ZT2 and ZT14 and given in gray values (mean gray value of the stained structure minus mean gray value of the background; see Material and Methods). In the $\mathrm{LN}_{\mathrm{v}}$ terminals, staining intensity was significantly higher at ZT2 than at ZT14 independently of the strain (two-way ANOVA, $F_{(1,54)}=58.912, p<$ 0.0001 for time; $F_{(2.54)}=0.588, p=0.559$ for strains $)$. In the terminals of neuron 8 (line Mz1525-gal4; UAS-pdf), no significant difference in staining difference was found at either time points (ANOVA, $F_{(1,15)}=0.660$, $p=0.429)$.

PDF accumulation was observed, leading perhaps to a constant release of PDF from the nerve terminals.

\section{DISCUSSION}

The aim of the present study was to investigate the function of the neuropeptide PDF in the circadian system of D. melanogaster. Former studies have revealed PDF in a well defined subset of neurons that express the clock genes per, tim, and $d b t$ and are most likely circadian pacemaker neurons (for review, see Kaneko, 1998; Hall, 1998). PDF was so far used as a marker molecule for these cells (Helfrich-Förster and Homberg, 1993; HelfrichFörster, 1995, 1997, 1998). Here we show that PDF by itself may serve as a chronobiological signaling substance in D. melanogaster as was already known for crabs (Aréchiga et al., 1985) and more recently also demonstrated for cockroaches (Petri and Stengl, 1997). Misexpression of the $p d f$ gene in the nervous system of Drosophila led to severe alterations in eclosion and activity rhythms. These rhythm alterations were observed in three of the eight tested lines and appeared to be correlated with the presence of "extra" PDF in the dorsal protocerebrum (see Action site of PDF). All three lines showed very similar changes in the activity pattern. There was an extension of activity into the dark phase of the LD cycle, an increase in activity level under DD conditions, and a destabilization of the activity rhythm evident in the frequent occurrence of complex activity patterns and arrhythmicity.

Although additional PDF affected behavioral rhythmicity severely, it did not lead to a complete arrhythmicity comparable to that of the known clock-gene rhythm mutants or the anatomical brain mutant disconnected, which lacks the LN (for review, see Hall, 1998). This might be attributable partly to the fact that PDF is still rhythmically accumulated in the $\mathrm{LN}_{\mathrm{v}}$ terminals of the flies with ectopic PDF. Probably PDF is also still rhythmically released from these terminals (see below), leading to a mixture of rhythmic and arrhythmic signals in the dorsal protocerebrum, a fact that could explain to some degree the complex rhythmicity of the flies.

Furthermore, we did not eliminate other possible circadian mediator molecules that might be used as output signals in these neurons. In the accessory medulla of other insects as well as in the SCN of mammals, several neuropeptides were colocalized in the same cells (Reghunandanan et al., 1993; Würden and Homberg, 1995), and all could function as circadian mediators. In the SCN, vasopressin is the most abundant neuropeptide and is thought to play a crucial role in the circadian system (Inouye, 1996; Jin et al., 1999). Nevertheless, rats without any vasopressin show astonishingly normal rhythms, although some rhythms had a reduced amplitude (Reppert et al., 1987; Brown and Nunez, 1989). Similarly, flies lacking PDF attributable to a nonsense mutation in the $p d f$ gene are not completely arrhythmic but still show initially weak rhythms with short period (Renn et al., 1999). In D. melanogaster, it is not known whether the $\mathrm{LN}_{\mathrm{v}} \mathrm{s}$ contain other neuropeptides in addition to PDF, but there are other per- and tim-expressing neurons that lack PDF, namely the $\mathrm{LN}_{\mathrm{d}}$ and $\mathrm{DN}$. These neurons contribute most likely to behavioral rhythmicity (Helfrich-Förster, 1998) and use neurotransmitters or neuromodulators that are different from those of the $\mathrm{LN}_{\mathrm{v}}$ and are not affected by PDF misexpression.

\section{Action site of PDF}

Our results showed that the action site of PDF is restricted to the central brain. We could not disturb the activity rhythm by ectopical expression of PDF in the thoracic NS, although some of these fibers even form an extensive plexus at the border between the thoracic NS and the blood and suggest a neurohormonal release of PDF. Neither could we affect the rhythm by expressing PDF in neurons associated with the corpora cardiaca, like the neurosecretory cells of the pars intercerebralis and pars lateralis. This indicates that PDF does not act as a neurohormone in the classical sense. Intriguingly, the existence of such a diffusible substance was predicted by brain transplantation experiments per- 
formed a long time ago (Handler and Konopka, 1979). Our results indicate that PDF is not the substance in question; they are more consistent with the assumption that PDF is a local neuromodulator that exerts its effect in the dorsal protocerebrum. Only two neurons with ectopic PDF expression (neuron 8 and 11) were common to all lines with abnormal rhythmicity but were not labeled in the ones with normal rhythmicity, suggesting that these cells are most likely responsible for the abnormal activity patterns. Both neurons projected into the dorsolateral protocerebrum, a brain region that receives the terminals of the natural PDF-producing $\mathrm{LN}_{\mathrm{v}} \mathrm{s}$ (Helfrich-Förster, 1997). The latter showed a cycling in PDH immunoreactivity. PDH immunoreactivity was high at ZT2 and low at ZT14. Opposed to that, PDH immunoreactivity was similarly low at both time points in the terminals of neuron 8.

High PDH immunoreactivity may indicate accumulation of PDF combined with little release from the terminals, whereas low PDH immunoreactivity may stand for a strong peptide release. If this is true, PDF should be constantly released from the neurites of neuron 8 and even at a higher constant level than those of neuron 11, because the neurites of neuron 11 were always very weakly labeled. This would lead to a permanent presence of PDF in the dorsolateral protocerebrum, a brain region that might be specially sensitive to PDF. Former observations have already shown that this brain region is crucial for the transfer of rhythmic signals from the $\mathrm{LN}_{\mathrm{v}}$ terminals via still unknown interneurons toward the locomotor centers (Helfrich-Förster, 1998). Therefore, it is conceivable that a constant release of PDF in addition to the rhythmic one of the $\mathrm{LN}_{\mathrm{v}} \mathrm{s}$ into this brain region will affect rhythmic behavior.

\section{Only specific cells are capable of PDF processing}

Like most neuropeptides, PDF of D. melanogaster is synthesized from a larger precursor composed of a signal peptide (16 aa), the PDF-associated peptide (63 aa), and PDF itself (18 aa) (Park and Hall, 1998). Such peptide precursors usually enter the Golgi apparatus where sorting and packaging into regulated secretory granules occurs according to conformation-dependent sorting signal motifs in the signal peptide (Loh et al., 1997) and where the precursor is processed into the mature peptide. Several enzymes are involved in this process: carboxypeptidases (CPEs), prohormone convertases, and a peptidylglycine $\alpha$-amidating monooxygenase (Fricker, 1988; Kolhekar et al., 1997; Udupi et al., 1997). The polyclonal antiserum against crab PDH that we used for detection of PDF appears to be directed against the amidated biological active $\mathrm{C}$ terminus of the peptide (H. Dircksen, personal communication) as well as against a putative second epitope present in the PDF sequence because this antiserum has been successfully used in a sandwich-ELISA, which usually requires the existence of two different epitopes (Löhr et al., 1993). It recognizes the mature PDHs and PDFs of all crabs and insects studied so far, but it seems not to recognize the precursors. We detected such mature PDF only in a subset of the expected PDF-positive cells in the different UAS-pdf;gal4-lines, whereas $p d f$ mRNA was present in all expected cells. This indicates that the mature peptide is not produced in every cell, although the $p d f$ gene is transcribed and probably also translated into the precursor peptide in the relevant cell. This holds true also if one uses UAS- $p d f$ lines carrying the $p d f$ gene from $D$. melanogaster instead of the $p d f$ gene from the grasshopper $R$. microptera (M. Täuber, unpublished results) that was used here. Obviously, D. melanogaster can process the $R$. microptera PDF precursor peptide like its own, but not all cells have the enzymatic capability for this processing.

The effects on rhythmic behavior were also very similar between ectopic Drosophila PDF and Romalea PDF, pointing to an evolutionary well conserved function of PDF (M. Täuber, C. Helfrich-Förster, unpublished results). Drosophila and Romalea PDF differ in 4 of the 18 amino acids (Park and Hall, 1998).

\section{Rhythmic control of PDF occurs post-translationally}

The $p d f$ gene appears not to be a clock-controlled gene as opposed to other genes with still unknown function that show a clock gene-dependent circadian fluctuation in the amount of mRNA (Van Gelder and Krasnow, 1996; Rouyer et al., 1997). Levels of $p d f$ mRNA were constant throughout the circadian cycle and not influenced in the arrhythmic mutant per $^{0}$ (Park and Hall, 1998). Nevertheless, PDF seems to be controlled post-translationally in a circadian manner: the rhythm in PDF accumulation in the central brain terminals was absent in the arrhythmic mutants $\mathrm{per}^{0}$ and tim $^{0}$ (Park et al., 2000). This suggests that PDF is not rhythmically produced but rather transported and/or secreted in a rhythmical manner and that this transport (and/or secretion) is somehow dependent on the clock genes per and tim. Alternatively, PDF precursor processing might involve clockcontrolled steps. One or more of the involved enzymes may be regulated in a circadian manner. Interestingly, CPE expression pattern shows a remarkable reaction to dark-to-light transition in the rat retina (Schlamp and Nickells, 1996). CPE is strongly expressed in photoreceptors in the dark. During light onset, CPE expression is rapidly induced in retinal ganglion cells, whereas expression in photoreceptors is reduced. This pattern is reversed when the animals are reexposed to the dark. Whether this process is clockcontrolled or just light dependent remains elusive.

Overexpression of the $p d f$ gene in the pacemaker cells did lead to a subjectively higher PDH immunoreactivity in the somata of the cells but not to a continuous accumulation of PDF in the central brain neurites. This indicates that the transport of PDF into the neurites or the secretion of PDF from nerve terminals is clock-controlled rather than PDF processing. In the ectopically $p d f$-expressing cells that lack the clock genes, these circadian regulatory mechanisms may be absent, and consequently we did not observe a rhythmical PDF accumulation in their nerve terminals.

Further studies are necessary to clarify how the clock genes per and tim are involved in processing, transport, or even secretion of PDF in D. melanogaster. Several mechanisms may contribute to rhythmical control of PDF.

\section{Putative role of PDF}

According to our results, PDF is most probably a local neuromodulator that exerts its effect in the dorsal protocerebrum. From their results with local injections of PDF into the accessory medulla of the cockroach, Petri and Stengl (1997) concluded that PDF acts as an inhibitory neuromodulator. We found a significant elevation of the activity level in flies with ectopic PDF, suggesting that PDF raises the excitability of downstream neurons, but it could also inhibit the activity of neurons that have an inhibiting effect on the overall arousal. Furthermore, the experiments on cockroaches point to a role of PDF in the input pathway rather than in the output pathway of the clock. Here, we favor the hypothesis that PDF is involved in the output pathway of the clock, although we cannot exclude the possibility that PDF additionally acts as input factor. Similar to the cockroach, the large 
PDF cells project contralaterally and may contact their siblings in the contralateral accessory medulla. Possibly PDF is used as a neuropeptide transmitter synchronizing the pacemaker cells of both brain hemispheres. A disturbed internal synchronization caused by PDF misexpression may effect behavioral rhythms as does a disturbed clock output. However, we did not observe an internal desynchronization in the PDF accumulation in the $\mathrm{LN}_{\mathrm{v}}$ central brain terminals of both hemispheres. Therefore, we think that the observed effects on behavioral rhythmicity are more likely attributable to action of PDF on the clock's output pathway. Further studies are necessary to reveal the specific function of PDF in the circadian system of insects.

\section{REFERENCES}

Albers HE, Liou S-Y, Stopa EG, Zoeller RT (1991) Interaction of colocalized neuropeptides: functional significance in the circadian timing system. J Neurosci 11:846-851.

Albers HE, Liou S-Y, Stopa EG, Zoeller RT (1992) Neurotransmitter colocalization and circadian rhythms. Prog Brain Res 92:289-307.

Aréchiga H, Cortes JL, Farcia U, Rodrigues-Sosa L (1985) Neuroendocrine correlates of circadian rhythmicity in Crustaceans. Am Zool 25:265-274.

Brand A (1995) GFP in Drosophila. Trends Genet 11:324-325.

Brand AH, Perrimon N (1993) Targeted gene expression as a means of altering cell fates and generating dominant phenotypes. Development 118:401-415.

Brand AH, Manoukian AS, Perrimon N (1994) Ectopic expression in Drosophila. Methods Cell Biol 44:635-654.

Brown MH, Nunez AA (1989) Vasopressin-deficient rats show a reduced amplitude of the circadian sleep rhythm. Physiol Behav 46:759-762.

Dircksen H, Zahnow CA, Gaus G, Keller R, Rao KR, Riehm JP (1987) The ultrastructure of nerve endings containing pigment-dispersing hormone $(\mathrm{PDH})$ in crustacean sinus glands: identification by an antiserum against synthetic PDH. Cell Tissue Res 250:377-387.

Dowse HB, Ringo JM (1987) Further evidence that the circadian clock in Drosophila is a population of coupled ultradian oscillators. J Biol Rhythms 2:65-76.

Dunlap JC (1999) Molecular bases for circadian clocks. Cell 96:271-290.

Dushay MS, Konopka RJ, Orr D, Greenacre ML, Kyriacou CP, Rosbash M, Hall JC (1990) Phenotypic and genetic analysis of clock, a new circadian rhythm mutant in Drosophila melanogaster. Genetics 125:557-578.

Fischer JA, Giniger E, Maniatis T, Ptashne M (1988) GAL4 activates transcription in Drosophila. Nature 332:853-865.

Freeman M (1996) Reiterative use of the EGF-receptor triggers differentiation of all cell-types in the Drosophila eye. Cell 87:651-660.

Fricker LD (1988) Carboxypeptidase E. Annu Rev Physiol 50:309-321. Glaser WR (1978) Varianzanalyse. Stuttgart: Gustav Fischer Verlag.

Hall JC (1998) Molecular neurogenetics of biological rhythms. J Neurogenet 12:115-181.

Handler AM, Konopka RJ (1979) Transplantation of a circadian pacemaker in Drosophila. Nature 279:236-238.

Hanesch U, Fischbach K-F, Heisenberg M (1989) Neuronal architecture of the central complex in Drosophila melanogaster. Cell Tissue Res 257:343-366

Helfrich-Förster C (1995) The period clock gene is expressed in central nervous system neurons which also produce a neuropeptide that reveals the projections of circadian pacemaker cells within the brain of Drosophila melanogaster. Proc Natl Acad Sci USA 92:612-616.

Helfrich-Förster C (1997) Development of pigment-dispersing hormone immunoreactive neurons in the nervous system of Drosophila melanogaster. J Comp Neurol 380:335-354.

Helfrich-Förster C (1998) Robust circadian rhythmicity of Drosophila melanogaster requires the presence of lateral neurons: a brain-behavioral study of disconnected mutants. J Comp Physiol [A] 182:435-453.

Helfrich-Förster C (2000) Differential control of morning and evening components in the activity rhythm of Drosophila melanogaster: sex specific differences suggest a different quality of activity. J Biol Rhythms, in press.

Helfrich-Förster C, Homberg U (1993) Pigment-dispersing hormoneimmunoreactive neurons in the nervous system of wild-type Drosophila melanogaster and of several mutants with altered circadian rhythmicity. J Comp Neurol 337:177-190.

Helfrich-Förster C, Stengl M, Homberg U (1998) Organization of the circadian system in insects. Chronobiol Int 15:567-594.

Hunter-Ensor M, Ousley A, Sehgal A (1996) Regulation of the Drosophila protein timeless suggests a mechanism for resetting the circadian clock by light. Cell 84:677-685.

Inouye S-IT (1996) Circadian rhythms of neuropeptides in the suprachiasmatic nucleus. In: Progress in brain research (Buis RM, Kalsbeek A, Romijn HJ, Pennartz CMA, Mirmiran M, eds) pp 75-90. New York: Elsevier.

Ito K, Sass H, Urban J, Hofbauer A, Schneuwly S (1997) GAL4responsive UAS-tau as a tool for studying the anatomy and development of the Drosophila central nervous system. Cell Tissue Res 290:1-10.

Jin X, Shearman LP, Weaver DR, Zylka MJ, DeVries GJ, Reppert SM (1999) A molecular mechanism regulating rhythmic output from the suprachiasmatic circadian clock. Cell 96:57-68.

Kaneko M (1998) Neural substrates of Drosophila rhythms revealed by mutants and molecular manipulations. Curr Opin Neurobiol 8:652-658.

Kaneko M, Helfrich-Förster C, Hall JC (1997) Spatial and temporal expression of the period and timeless genes in the developing nervous system of Drosophila: newly identified pacemaker candidates and novel features of clock gene product cycling. J Neurosci 17:6745-6760.

Klein DC, Moore RY, Reppert SM (1991) Suprachiasmatic nucleus. The mind's clock. New York: Oxford UP.

Kloss B, Price JL, Saez L, Blau J, Rothenfluh A, Wesley CS, Young MW (1998) The Drosophila clock gene double-time encodes a protein closely related to human casein kinase Ie. Cell 94:97-107.

Kolhekar AS, Roberts MS, Jiang N, Johnson RC, Mains RE, Eipper BA, Taghert PH (1997) Neuropeptide amidation in Drosophila: separate genes encode the two enzymes catalyzing amidation. J Neurosci 17:1363-1376.

Konopka RJ, Hamblen-Coyle MJ, Jamison C, Hall JC (1994) An ultrashort clock mutation at the period locus of Drosophila melanogaster that reveals some new features of the fly's circadian system. J Biol Rhythms 9:189-216.

Loh YP, Snell CR, Cool DR (1997) Receptor-mediated targeting of hormones to secretory granules: role of carboxypeptidase E. Trends Endocrinol Metab 8:130-137.

Löhr J, Klein J, Webster SG, Dircksen H (1993) Quantification, immunoaffinity purification and sequence analysis of a pigment-dispersing hormone of the shore crab Carcinus maenas (L.). Biochem Physiol [B] 104:699-706.

Nässel DR, Elekes K (1985) Serotonergic terminal in the neural sheath of the blowfly nervous system: electron microscopical immunocytochemistry and 5,7-dihydroxytryptamine labelling. Neuroscience 15:293-307.

Park JH, Hall JC (1998) Isolation and chronobiological analysis of a neuropeptide pigment-dispersing factor gene in Drosophila melanogaster. J Biol Rhythms 13:219-228.

Park JH, Helfrich-Förster C, Lee G-H, Liu L, Rosbash M, Hall JC (2000) Differential regulation of circadian pacemaker output by separate clock genes in Drosophila. Proc Natl Acad Sci USA, in press.

Petri B, Stengl M (1997) Pigment-dispersing hormone shifts the phase of the circadian pacemaker of the cockroach Leucophaea maderae. J Neurosci 17:4087-4093.

Petri B, Stengl M, Würden S, Homberg U (1995) Immunocytochemical characterization of the accessory medulla in the cockroach Leucophaea maderae. Cell Tissue Res 282:3-19.

Poeck B, Hofbauer A, Pflugfelder GO (1993) Expression of the Drosophila optomotor-blind gene transcript in neuronal and glial cells of the developing nervous system. Development 117:1017-1029.

Pyza E, Meinertzhagen IA (1996) Neurotransmitters regulate rhythmic size changes amongst cells in the fly's optic lobe. J Comp Physiol [A] 178:33-45.

Rao KR, Riehm JP (1993) Pigment-dispersing hormones. Ann NY Acad Sci 680:78-88.

Renn S, Park JH, Rosbash M, Hall JC, Taghert PH (1999) A pdf neuropeptide gene mutation and ablation of PDF neurons each cause severe abnormalities of behavioral circadian rhythms in Drosophila. Cell 99:791-802.

Reghunandanan V, Reghunandanan R, Singh PI (1993) Neurotransmit- 
ters of the suprachiasmatic nucleus: role in the regulation of circadian rhythms. Prog Neurobiol 41:647-655.

Reppert SM, Schwartz WJ, Uhl GR (1987) Arginine vasopressin: a novel peptide rhythm in cerebrospinal fluid. Trends Neurosci 10:76-80.

Robinow S, White K (1988) The locus elav of Drosophila melanogaster is expressed in neurons at all developmental stages. Dev Biol 126:294-303.

Robinow S, White K (1991) Characterization and spatial distribution of the ELAV protein during Drosophila melanogaster development. J Neurobiol 22:443-461.

Rouyer F, Rachidi M, Pikielny C, Rosbash M (1997) A new gene encoding a putative transcription factor regulated by the Drosophila circadian clock. EMBO J 16:3944-3954.

Schlamp CL, Nickells RW (1996) Light and dark cause a shift in the spatial expression of a neuropeptide-processing enzyme in the rat retina. J Neurosci 16:2164-2171.

Sokolove PG, Bushell WN (1978) The chi square periodogram: its utility for analysis of circadian rhythms. J Theor Biol 72:131-160.

Stengl M, Homberg U (1994) Pigment-dispersing hormone-immuno- reactive neurons in the cockroach Leucophaea maderae share properties with circadian pacemaker neurons. J Comp Physiol [A] 175:203-213.

Udupi V, Gomez P, Song L, Varlamow O, Reed JT, Leiter EH, Fricker LD, Greeley GH (1997) Effect of carboxypeptidase E deficiency on progastrin processing and gastrin messenger ribonucleic acid expression in mice with the fat mutation. Endocrinology 138:1959-1963.

Van Gelder RN, Krasnow MA (1996) A novel circadianly expressed Drosophila melanogaster gene dependent on the period gene for its rhythmic expression. EMBO J 15:1625-1631.

Würden S, Homberg U (1995) Immunocytochemical mapping of serotonin and neuropeptides in the accessory medulla of the locust, Schistocerca gregaria. J Comp Neurol 362:305-319.

Yao K-M, Samson M-L, Reeves R, White K (1992) Gene elav of Drosophila melanogaster: a prototype for neuronal-specific RNA binding protein gene family that is conserved in flies and humans. J Neurobiol 24:723-739.

Zar JH (1984) Biostatistical analysis. Englewood Cliffs, NJ: PrenticeHall. 